\title{
Flexible and Cellulose-based Organic Electronics
}

\author{
Jesper Edberg
}




\section{Flexible and Cellulose-based Organic Electronics}

Jesper Edberg

During the course of the research underlying this thesis, Jesper Edberg was enrolled in Agora Materiae, a multidisciplinary doctoral program at Linköping University, Sweden.

Linköping Studies in Science and Technology. Dissertations. No. 1845 (C)2017 Jesper Edberg unless otherwise noted Cover by Jesper Edberg Printed by LiU-Tryck, Linköping, Sweden, 2017 ISBN: 978-91-7685-542-3

ISSN: 0345-7524

Electronic publication: http://www.ep.liu.se 
"An expert is a person who has made all the mistakes that can be made in a very narrow field."

-Niels Bohr 



\section{Abstract}

Organic electronics is the study of organic materials with electronic functionality and the applications of such materials. In the 1970s, the discovery that polymers can be made electrically conductive led to an explosion within this field which has continued to grow year by year. One of the attractive features of organic electronic materials is their inherent mechanical flexibility, which has led to the development of numerous flexible electronics technologies such as organic light emitting diodes and solar cells on flexible substrates. The possibility to produce electronics on flexible substrates like plastic or paper has also had a large impact on the field of printed, electronics where inks with electronic functionality are used for large area fabrication of electronic devices using classical printing methods, such as screen printing, inkjet printing and flexography.

Recently, there has been a growing interest in the use of cellulose in organic and printed electronics, not only as a paper substrate but also as a component in composite materials where the cellulose provides mechanical strength and favorable 3D-microstructures. Nanofibrillated cellulose is composed of cellulose fibers with high aspect-ratio and diameters in the nanometer range. Due to its remarkable mechanical strength, large area-to-volume ratio, optical transparency and solution processability it has been widely used as a scaffold or binder for electronically active materials in applications such as batteries, supercapacitors and optoelectronics.

The focus of this thesis is on flexible devices based on conductive polymers and can be divided into two parts: (1) Composite materials of nanofibrillated cellulose and the conductive polymer PEDOT:PSS and (2) patterning of vapor phase polymerized conductive polymers. In the first part, it is demonstrated how the combination of cellulose and conductive polymers can be used to make electronic materials of various form factors and functionality. Thick, freestanding and flexible "papers" are used to realize electrochemical devices such as transistors and supercapacitors while lightweight, porous and elastic aerogels are used for sensor applications. The second focus of the thesis is on a novel method of patterning conductive polymers produced by vapor phase polymerization using UV-light. This method is used to realize flexible electrochromic smart windows with highresolution images and tunable optical contrast. 



\section{Populärvetenskaplig sammanfattning}

Organisk elektronik är studien av organiska material med elektronisk funktionalitet samt applikationer av sådana material. Upptäckten att polymerer kan göras elektriskt ledande på 1970-talet ledde till ett uppsving inom detta forskningsfält som har fortsatt att växa sedan dess. En av de attraktiva egenskaperna hos organiska material med elektronisk funktionalitet är deras naturliga mekaniska flexibilitet vilket har resulterat i många applikationer inom flexibel elektronik så som organiska ljus-emitterande dioder (OLED) och organiska solceller på flexibla substrat. Möjligheten att producera elektronik på flexibla substrat så som plast eller papper har också haft en stor inverkan på forskningsfältet "tryckt elektronik", där elektroniskt funktionaliserade bläck används för storskalig produktion av elektroniska komponenter och kretsar med hjälp av vanliga tryckmetoder så som serigrafi, bläckstråleskrivare och flexografi.

De senaste åren har det visats ett ökat intresse för att använda cellulosa inom organisk och tryckt elektronik, inte bara som ett papperssubstrat, utan också som en komponent i kompositmaterial där cellulosan bidrar med mekanisk styrka och en fördelaktig 3D-mikrostruktur. Nanofibrillär cellulosa är cellulosafiber med diameter i nanometerskala och en längd på flera mikrometer. Tack vare dess fantastiska mekaniska styrka, stora specifika area, optiska transparens och processbarhet i lösningsform så har den använts i applikationer så som batterier, superkondensatorer och optoelektronik.

Denna avhandling fokuserar på flexibel elektronik baserad på elektriskt ledande polymerer och kan delas in i två delar: (1) Kompositmaterial av nanofibrillär cellulosa och den ledande polymeren PEDOT:PSS och (2) mönstring av ledande polymerer producerade med metoden "vapor phase polymerization". I avhandlingens första fokus demonstreras det hur kombinationen av cellulosa och ledande polymerer kan användas för att producera material av olika former, strukturer och storlekar samt olika funktionaliteter. Tjocka, självupphållande och flexibla "pappersark" användes för att konstruera elektrokemiska komponenter så som transistorer och superkondensatorer medan lättviktiga, porösa och elastiska "aerogels" användes för sensorapplikationer. Det andra fokuset i avhandlingen handlar om en nyutvecklad mönstringsmetod för elektriskt ledande polymerer som producerats via "vapor phase polymerization" med hjälp av UV-ljus. Denna metod användes för att tillverka flexibla elektrokroma smarta fönster med hög upplösning och elektroniskt justerbar optisk kontrast. 



\section{Acknowledgements}

This work would not have been possible without the help and support of many people to whom I am sincerely grateful. I would like to give a special thanks to:

My supervisors, Magnus Berggren and Isak Engquist, for all their help, guidance, and encouragement.

The other seniors, Magnus Jonsson, Daniel Simon, Xavier Crispin, Eleni Stavrinidou, Igor Zozoulenko, Simone Fabiano, Roger Gabrielsson, and Eric Glowacki for many interesting discussions and collaborations.

Sophie Lindesvik, Åsa Wallhagen, Sandra Scott, and Katarina Swanberg for all the help with everything related to administration.

Lars Gustavsson, Putte Eriksson, and Anna Malmström for keeping the lab up and running.

Zia Ullah Khan and Simone Fabiano for teaching me how everything in the lab works and always taking the time to answer stupid questions.

Isak Engquist, Magnus Berggren, Robert Brooke, Zia Ullah Khan, Negar Sani, and Johannes Gladisch for helping me with reviewing my thesis.

The past and present members of the research school Agora Materia, and especially Per-Olof Holtz, for organizing interesting seminars, study visits, and conferences.

My "LOE-siblings", Robert Brooke, Donata Iandolo, Negar Sani, and Dan Zhao for all the laughs.

The whole LOE and Acreo team for all the good times and fun collaborations.

My family, for always encouraging me to walk my own path.

Henric Thornér, for all the love and support, and for keeping me mentally stable during the writing of this thesis. 



\section{List of Included Papers}

\section{Paper I:}

\section{An Organic Mixed Ion-Electron Conductor for Power Electronics}

Abdellah Malti†, Jesper Edberg', Hjalmar Granberg, Zia Ullah Khan, Jens W. Andreasen, Xianjie Liu, Dan Zhao, Hao Zhang, Yulong Yao, Joseph W. Brill, Isak Engquist, Mats Fahlman, Lars Wågberg, Xavier Crispin and Magnus Berggren.

Advanced Science, 2015, 3(2), 1500305.

† These authors contributed equally to this work.

Contribution: Large parts of development, manufacturing and characterization of materials and devices. Wrote large parts of the first draft and contributed to the final editing of the manuscript.

\section{Paper II:}

\section{Thermoelectric Polymers and their Elastic Aerogels}

Zia Ullah Khan, Jesper Edberg, Mahiar Max Hamedi, Roger Gabrielsson, Hjalmar Granberg, Lars Wågberg, Isak Engquist, Magnus Berggren and Xavier Crispin.

Advanced Materials, 2016, 28(22), 4556-4562.

Contribution: Large parts of development, manufacturing and characterization of materials and devices used for flexible dual sensors. Wrote parts of the first draft and contributed to the final editing of the manuscript.

\section{Paper III:}

Electrochemical Circuits from "Cut and Stick" PEDOT:PSS-nanocellulose Composite (Submitted)

Jesper Edberg, Abdellah Malti, Hjalmar Granberg, Mahiar Max Hamedi, Xavier Crispin, Isak Engquist and Magnus Berggren.

Contribution: All experimental work. Wrote the first draft and contributed to the final editing of the manuscript. 


\section{Paper IV:}

\section{Boosting the Capacity of All-Organic Paper Supercapacitors Using Wood Derivatives (Manuscript)}

Jesper Edberg, Olle Inganäs, Isak Engquist, Magnus Berggren.

Contribution: All experimental work. Wrote the first draft and contributed to the final editing of the manuscript.

\section{Paper V:}

Patterning and Conductivity Modulation of Conductive Polymers by UV Light Exposure

Jesper Edberg, Donata Iandolo, Robert Brooke, Xianjie Liu, Chiara Musumeci, Jens Wenzel Andreasen, Daniel T. Simon, Drew Evans, Isak Engquist and Magnus Berggren.

Advanced Functional Materials, 2016, 26(38), 6950-6960.

Contribution: Large part of the development and investigation of the patterning technique. Wrote majority of the first draft and contributed to the final editing of the manuscript.

\section{Paper VI}

Complementary Polymer Electrochromic Devices Enabling Dynamic Change Between two High-resolution Images (Submitted)

Robert Brooke, Jesper Edberg, Donata Iandolo, Magnus Berggren, Isak Engquist, Xavier Crispin.

Contribution: Participated in the design of the devices. Sample preparation and characterization of color contrast and electrochemical properties. Wrote parts of the first draft and contributed to the final editing of the manuscript. 


\section{Related Work Not Included in the Thesis}

A novel investigation on carbon nanotube/ $\mathrm{ZnO}, \mathrm{Ag} / \mathrm{ZnO}$ and $\mathrm{Ag} /$ carbon nanotube/ZnO nanowires junctions for harvesting piezoelectric potential on textile

Azam Khan, Jesper Edberg, Omer Nur and Magnus Willander.

Journal of Applied Physics, 2014, 116(3), 034505.

Frequency-Dependent Photothermal Measurement of Transverse Thermal Diffusivity of Organic Semiconductors

J. W. Brill, Maryam Shahi, Marcia M. Payne, Jesper Edberg, Y. Yao, Xavier Crispin and J. E. Anthony.

Journal of Applied Physics, 2015, 118(23), 235501. 



\section{Acronyms}

AFM atomic force microscopy

CMC carboxymethyl cellulose

CNT carbon nanotube

ECD electrochromic device

EDLC electric double layer capacitor

EIS electrochemical impedance spectroscopy

emf electromotive force

ESR equivalent series resistance

FET field effect transistor

ITO indium tin oxide

LIB lithium ion battery

LS lignosulfonate

NHE normal hydrogen electrode

OFET organic field effect transistor

EGOFET electrolyte gated organic field effect transistor

OECT organic electrochemical transistor

PANI polyaniline

PDMS polydimethylsiloxane

PEDOT poly(3,4-ethylenedioxythiophene)

PPy polypyrrole

PSS poly(styrene sulfonate)

SCE standard calomel electrode

TEG thermoelectric generator

Tos tosylate or p-toluenesulfonic acid

VPP vapor phase polymerization 



\section{Table of Contents}

\section{Part I: Background}

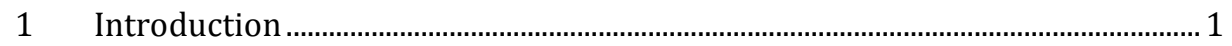

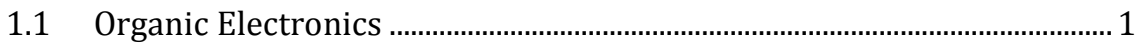

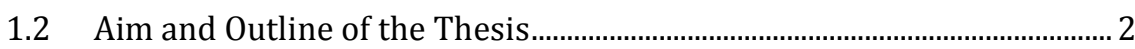

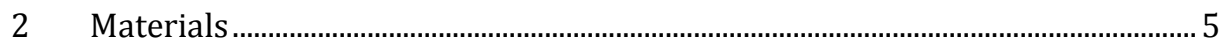

2.1 Conjugated Polymers.................................................................................... 5

2.1.1 Atomic and Molecular Orbitals....................................................... 5

2.1.2 Electronic Structure ………………………………………………..... 7

2.1.3 Doping and Charge Carriers...............................................................

2.1.4 Charge Transport and Disorder .....................................................12

2.1.5 PEDOT ...............................................................................................14

2.2 Cellulose ................................................................................................

2.2.1 Wood Fiber Structure ……………………………………………....17

2.2.2 Nanocellulose ...............................................................................18

2.2.3 Nanofibrillated Cellulose..................................................................19

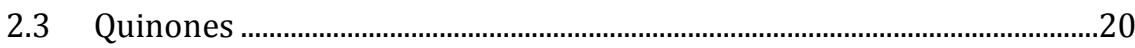

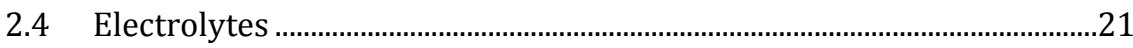

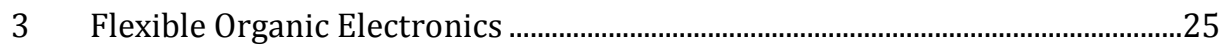

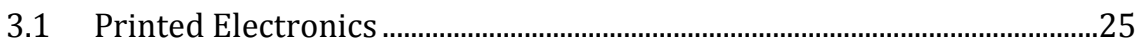

3.2 Photopatterning of Conductive Polymers ………………………….............25

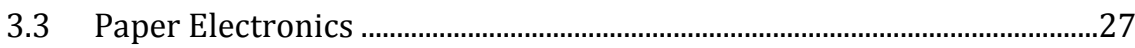

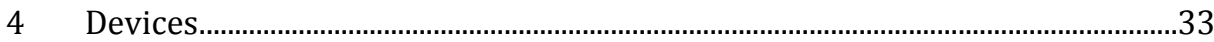

4.1 Capacitors ...................................................................................................33

4.1.1 Capacitance .................................................................................33

4.1.2 The Electric Double Layer................................................................36

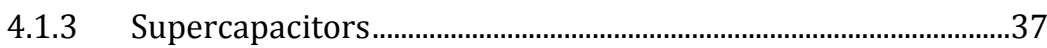

4.1.4 Hybrid Supercapacitors .......................................................................39

4.2 Organic Electrochemical Transistors ……………………..........................41

4.3 Thermoelectric Generators...............................................................................4

4.3.1 The Seebeck Effect ................................................................................ 44

4.3.2 Energy Harvesting with TEGs ........................................................45

4.3.3 Dual Sensors ...................................................................................46

$4.4 \quad$ Electrochromic Devices.....................................................................................

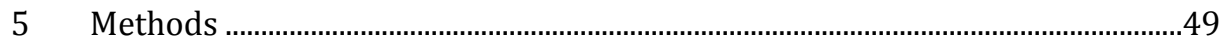


5.1 Solvent Casting and Spin Coating.................................................................4

5.2 Vapor Phase Polymerization............................................................................4

$5.3 \quad$ Freeze-drying .........................................................................................

5.4 Electrochemical Characterization................................................................52

5.4.1 The Electrochemical Cell ..............................................................52

5.4.2 Cyclic Voltammetry..........................................................................5

5.4.3 Chronopotentiometry ...............................................................56

5.4.4 Impedance Spectroscopy ………………………......................57

5.5 Conductivity Measurements...........................................................................60

5.6 Absorption Spectroscopy...........................................................................61

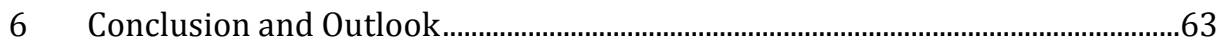

6.1 NFC-PEDOT:PSS Composites.......................................................................63

6.2 Patterning Conductive Polymers with UV Light............................................64

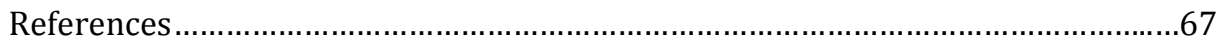

Part II: Papers 
Part I

Background 



\section{Introduction}

\subsection{Organic Electronics}

Organic electronics is the study of the electronic properties of certain organic (carbon-based) materials as well as the development of electronic devices and circuits based on such materials. This field of research dates back to the $19^{\text {th }}$ century with the discovery of the first conductive polymer, polyaniline, by Henry Letheby. His findings were published in a paper titled "On the production of a blue substance by the electrolysis of sulphate of aniline" in 1862.[1] However, it would take more than a century before the field of organic electronics would truly take off. In 1977, it was discovered by Hideki Shirakawa, Alan G. McDiarmid, and Alan J. Heeger that a highly conductive polymer could be produced by doping transpolyacetylene with halogen vapor. ${ }^{[2]}$ In 2000 they were awarded the Nobel prize in chemistry for their discovery. Since then, a large number of conductive polymers such as polypyrrole and polythiophene have been synthesized.

Classical electronics is largely based on inorganic materials like metals and silicon. These materials are hard, require high temperatures for processing and devices operate by electrical currents. Organic electronic materials on the other hand consist of molecules and polymers with electronic functionality. These materials can be soft and flexible, can be processed at room temperature in solution form and can conduct both electronic and ionic currents. These differences in material properties are the motivation for manufacturing organic electronic devices. The mechanical flexibility of organic materials has made possible a number of flexible electronics applications such as bendable OLED displays and organic solar cells on flexible substrates. Low temperature and solution processability of some organic conductors have been utilized in the field of printed electronics. Finally, the ability of some organic materials to conduct both electrons and ions make them ideal candidates for interfacing electronics with biological systems, so called bioelectronics. This is because biological organisms use ions and chemicals to communicate and regulate their functions rather than electrons. Furthermore, since these biological systems consist of soft materials, classical "hard" electronics are unsuited to use for such interfaces. 


\subsection{Aim and Outline of the Thesis}

The aim of this thesis is to develop new functional materials and patterning techniques for flexible organic electronics. The current way of manufacturing flexible electronics is to add functional materials on top of a flexible carrier substrate, such as paper or plastic sheets. The thickness of the active devices is typically $100 \mathrm{~nm}-10 \mu \mathrm{m}$ while the substrate is often in the order of $100 \mu \mathrm{m}$. This means that the carrier constitutes the majority of the weight and volume of the flexible devices. To exploit this untapped volume, substrates can be functionalized with active materials to realize bulk electronic devices. For this purpose, cellulose is preferred over synthetic polymer substrates due to its intrinsic porous structure and compatibility with many organic materials such as conductive polymers. Most of the scientific work in this thesis deals with the development and implementation of functional bulk materials based on cellulose and organic conductive materials.

Besides developing new materials, new methods of patterning organic electronic devices used for flexible electronics are of great importance to get around issues with existing techniques and to explore new manufacturing possibilities. With that motivation, the second focus of this thesis is to develop new patterning methods which solve some of the issues with existing technologies, as well as to introduce completely new functionality.

The first part of the thesis gives the necessary background to understand the theory, methodology and results of the scientific work. Chapter 2 deals with the most important materials used and introduces the theory of conductive polymers. Chapter 3 gives an overview of flexible organic electronics and the usage of cellulose and paper in this field. In Chapter 4 , the different devices which were constructed in the scientific work is presented. The most important methods of manufacturing and characterizing materials and devices used in the papers are presented in Chapter 5. Finally, Chapter 6 summarizes the scientific results and gives an outlook to future work.

In the second part of the thesis, the results of the scientific work are presented in six papers. Papers I-IV deal with composite materials of nanocellulose and the conductive polymer PEDOT:PSS. These materials are manufactured in the form of paper-like films as well as porous and lightweight aerogels which were used for various device applications including transistors, supercapacitors and sensors. Papers V and VI introduce a novel method of pattering conductive polymers produced by vapor phase polymerization. This method was further used to manufacture electrochromic devices with high resolution images and 
electronically tunable optical contrast, produced on both flexible and rigid substrates. 


\section{Materials}

\subsection{Conjugated Polymers}

Polymers are long molecules that consist of repeated molecular units. The word polymer is derived from the Greek words "many" (poly) and "parts" (mer). In their free form, the building blocks which makes up a polymer are called monomers. Polymers can be found in nature and are a crucial part of the bulk of all living matter. In plants, the most abundant molecule is cellulose which consists of repeating glucose molecules and belong to a class called polysaccharides. In animals, the proteins in our muscles and the DNA in the core of our cells are polymers and belong to the group of polypeptides and polynucleotides, respectively. Plastics, such as polyester and polyethylene terephthalate (PET), are examples of synthetic polymers. The use of plastics and other synthetic polymers has revolutionized our society, in many respects, during the last century.

Polymers are generally electrical insulators, and for a long time it was believed that they could never conduct electricity. However, with a special structure of the polymer backbone, semiconducting behavior can be achieved. With chemical or electrochemical doping, these polymers can be made highly conductive.

\subsubsection{Atomic and Molecular Orbitals}

Most polymers are organic materials, meaning that they have a backbone structure consisting of carbon atoms. With its four valence electrons, carbon can form many bonds with other atoms. Carbon has four atomic orbitals which can be occupied by electrons, one s-orbital and three p-orbitals ( $\mathrm{p}_{\mathrm{x}}, \mathrm{p}_{\mathrm{y}}$ and $\mathrm{p}_{\mathrm{z}}$ ). In its ground state configuration, two electrons reside in the s-orbital and two electrons in two of the p-orbitals, leaving one p-orbital unoccupied. The ground state configuration of carbon can thus be written as $1 s^{2} 2 s^{2} 2 p^{2}$ where the number before the orbital represent the energy level and the exponent gives the number of electrons in the specific orbital. $1 \mathrm{~s}^{2}$ are the two core electrons which don't generally have any influence on the chemistry and chemical bonds of carbon. Figure 2.1a shows the shape of the s- and p-orbitals, while Figure 2.1b and Figure $2.1 \mathrm{c}$ show their positions in a coordinate system. Figure $2.1 \mathrm{~d}$ shows the full set of orbitals together. 


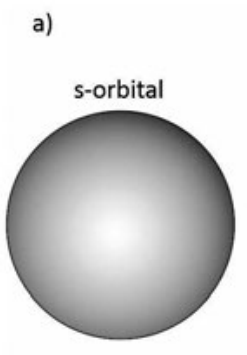

c)

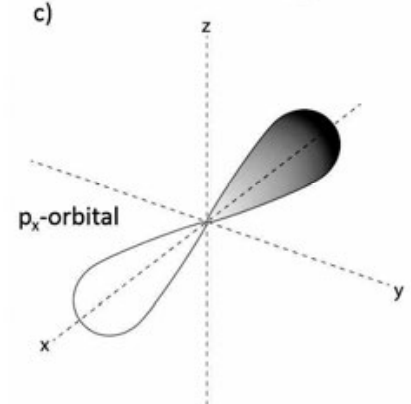

b)

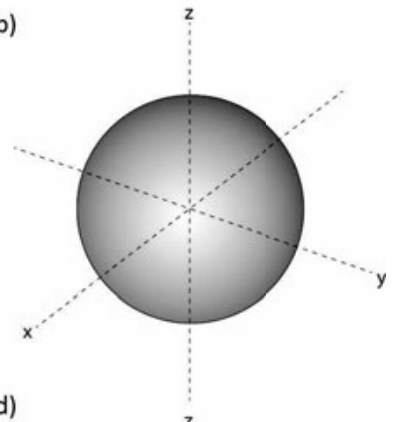

d)

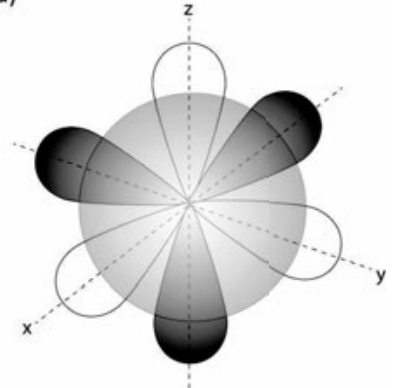

Figure 2.1 Atomic orbitals of unhybridized carbon.

By promoting one of the electrons in the 2s-orbital to the 2 p-orbital, several new atomic orbitals can be constructed by linear combinations. This process is referred to as hybridization and is the root for many of the bond configurations of carbon while forming molecules and polymers. The three hybridized states are called sp, $\mathrm{sp}^{2}$ and $\mathrm{sp}^{3}$. In sp-hybridization, the $2 \mathrm{~s}$ orbital has hybridized with one of the $2 \mathrm{p}$ orbitals, leaving two p-orbitals unchanged. Similarly, in $\mathrm{sp}^{2}$ - and $\mathrm{sp}^{3}$ hybridization, the s-orbital has hybridized with two and three of the p-orbitals respectively. Figure 2.2 shows the electronic configurations of the different hybridized states as well as the resulting shape of the $\mathrm{sp}^{2}$-state. In this state, the $\mathrm{sp}^{2}$-orbitals are oriented in a plane with $120^{\circ}$ angular separation. The

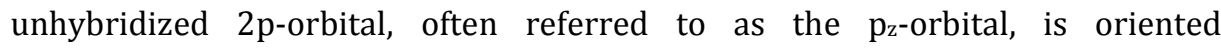
perpendicular to the plane (z-direction in Figure 2.2). In conductive polymers, it is the $\mathrm{sp}^{2}$-hybridization that gives rise to the electronic conduction. 

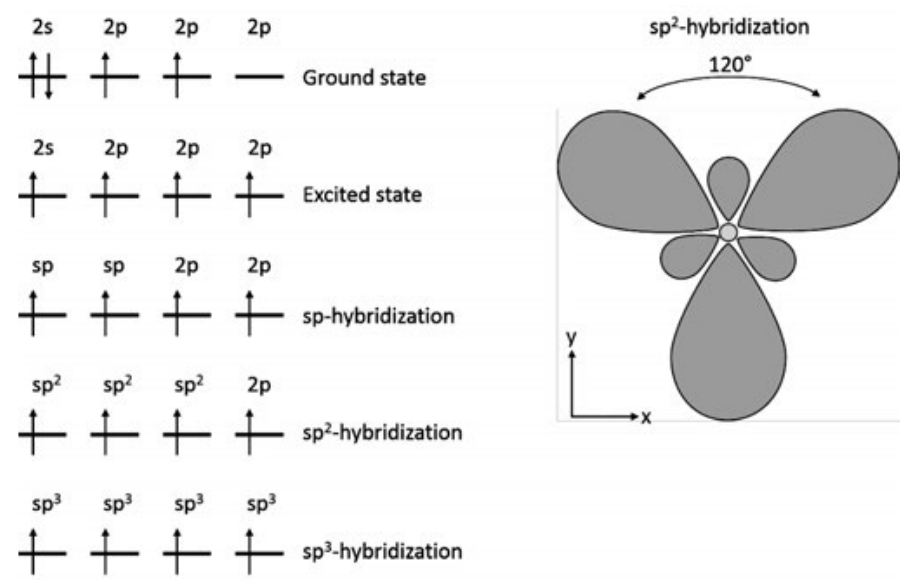

Figure 2.2 Hybridized states of carbon (left) and the structure of the $\mathrm{sp}^{2}$-orbitals. The black arrows in the state diagrams represent electrons with up- or down spin.

\subsubsection{Electronic Structure}

When two $\mathrm{sp}^{2}$-hybridized carbon atoms are brought in close proximity, their orbitals can overlap to form a covalent bond. This type of bond, which is called a $\sigma$ bond, make up the backbone structure of many organic materials. This is the case for the simple molecule ethene, which has two carbon atoms and four hydrogen atoms. The two $\mathrm{p}_{\mathrm{z}}$-orbitals of the two carbon atoms will also overlap and form a so called $\pi$-bond. The electrons in the overlapping $\mathrm{p}_{\mathrm{z}}$-orbitals are less localized than the electrons in the $\sigma$-bond and can therefore move from one site to the other. It is this type of delocalization of the electron wave functions that give rise to the electronic conductivity in polymers.

The overlap of the two orbitals generates two new molecular orbitals (MO) and two new energy levels for the electrons, since their wave functions can be combined in two different ways. The relatively lower energy state is called the bonding state and the higher energy is called the antibonding state. In the bonding state, there is constructive interference between the wave functions, resulting in a high electron density between the carbon atoms. The high electron density screens the positive charge of the atomic nuclei and thus lowers the overall energy of the molecule by reducing the electrostatic repulsion between the cores. Conversely, in the antibonding state there is destructive interference between the wave functions resulting in a low electron density between the nuclei and, thus, the energy of the resulting molecule is increased. The formation of the new molecular orbitals is illustrated in Figure 2.3 where two wave functions ( $\psi_{\mathrm{A}}$ and $\psi_{\mathrm{B}}$ ) are combined in a 
constructive and a destructive way, respectively. Since two electrons can occupy the same state, both the $\mathrm{p}_{\mathrm{z}}$-electrons will occupy the bonding state in the ground state to minimize the energy of the molecule.

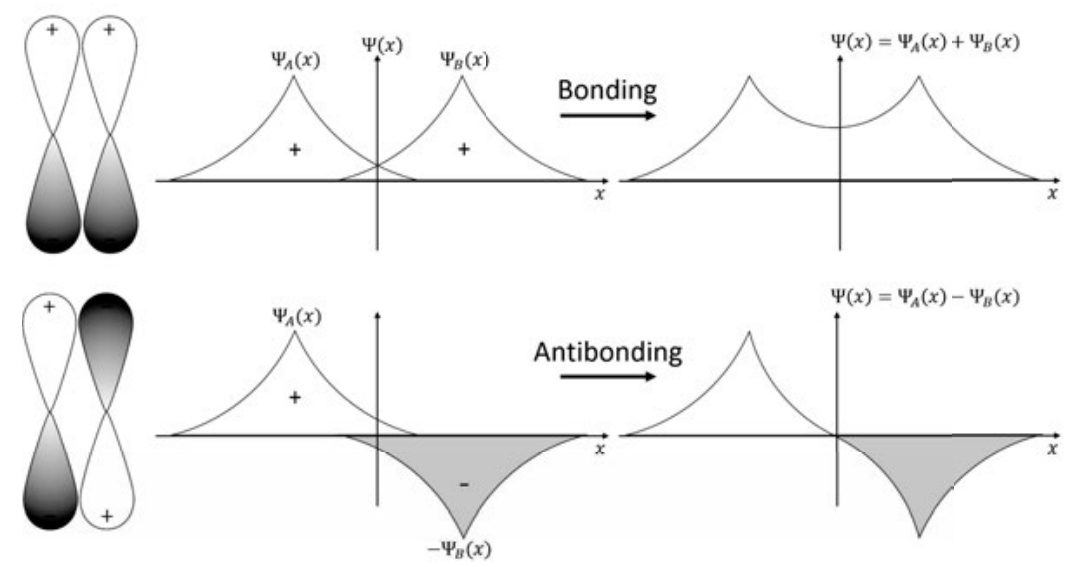

Figure 2.3 Bonding and antibonding orbitals.

By adding more carbon atoms to the chain, more orbitals will overlap to form new molecular orbitals. This can be represented by the polymer transpolyacetylene which has the repeat unit $\left[\mathrm{C}_{2} \mathrm{H}_{2}\right]_{\mathrm{n}}$. For each added repeat unit, two more carbon atoms are added and therefore two new orbitals with corresponding energy levels are formed. These types of polymers with delocalized electron states are called conjugated polymers. In the ground state configuration, the energy levels are half-occupied by electrons. The energy difference between the highest occupied molecular orbital (HOMO) and the lowest unoccupied molecular orbital (LUMO) is termed the band gap $\left(E_{g}\right)$. As the number of repeating units goes towards infinity, the discrete energy levels form a continuous band. However, due to a phenomenon called Peierls distortion, a gap is formed in the middle of the band. This gap is caused by a reorganization of the molecular bonds leading to alternating long and short bonds. Below the gap, all states are filled and above the gap all states are empty in the ground state of the polymer. The evolution of the energy bands of trans-polyacetylene is illustrated in Figure 2.4. In this picture, the leftmost energy level corresponds to a single $\mathrm{p}_{\mathrm{z}}$ atomic orbital. In a repeat unit of the polymer, which contain two carbon atoms, the energy levels split into two. Two repeat units, and four carbon atoms, results in four energy levels, and so on. 
Trans-polyacetylene<smiles>CC=CC=CC=CC=CC=CC=CC</smiles>

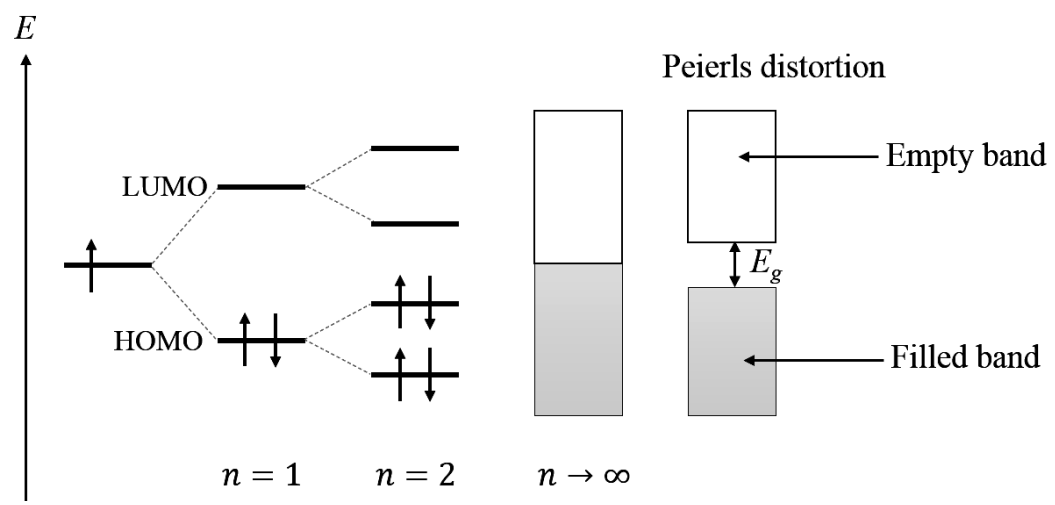

Figure 2.4 The formations of energy bands in trans-polyacetylene.

\subsubsection{Doping and Charge Carriers}

Conducting polymers, like trans-polyacetylene, are semiconductors with a bandgap around 1-4 eV. In their ground state configuration, semiconductors have no conductivity since the energy levels below the HOMO level are filled and the energy levels above the LUMO level are empty. This corresponds to a semiconductor at $0 \mathrm{~K}$. However, at temperatures above absolute zero, electrons in the valence band (band below HOMO) can be thermally excited to the conduction band (band above LUMO) where charge transport takes place. Therefore, as opposed to metals, the conductivity of an intrinsic semiconductor increases with temperature.

Even at high temperatures, intrinsic semiconductors have rather low conductivity. To achieve high enough conductivity to be useful in electronics applications, a process called doping is required. In inorganic semiconductors, doping involves the introduction of an impurity species into the lattice points of the material. These impurities act as electron donors or acceptors which introduces new conduction bands with mobile charge carriers. Very small amounts of impurities can increase the conductivity by several orders of magnitude in the case of inorganic semiconductors. Similarly, organic semiconductors also require doping to increase their electronic conductivity. However, the doping in this case involves oxidation or reduction of the polymer, with a charge transfer reaction between the polymer and dopant. To balance the new charge on the polymer, 
counterions are added which bond to the polymer. The ions can be small mobile ions, like chloride, or large immobile polyelectrolytes, like polystyrene sulfonate (PSS).

Polymers which are doped by oxidation are referred to as p-type since the charge carriers have a positive charge. Similarly, polymers doped by reduction are called n-type. Most conductive polymers reported in literature are p-type since they are typically more chemically stable in ambient atmosphere. When a charge (positive or negative) is added to the polymer, this results in a reorganization of the carbon bonds. This effect is strongest close to the charge but extends along the polymer. The charge and its resulting lattice deformation form a quasi-particle which is referred to as a soliton in case of a degenerate ground state energy and a polaron in case of nondegenerate ground state energy. ${ }^{[3]}$ Trans-polyacetylene is an example of a polymer with a degenerate ground state energy. This is because there are no energetically preferred sites for the alternating double end single bonds due to the symmetric structure of the polymer. However, most conductive polymers, like polythiophenes and polypyrrole, have a nondegenerate ground state. The addition of solitons or polarons change the band structure of the polymer and introduce new energy levels in the band gap of the intrinsic polymer. In polyheterocycles, the two possible arrangements of the single and double bonds (quinoid and benzenoid form) have different energy, resulting in two new energy levels. This results in a new band gap which is considerably smaller than that of an intrinsic semiconductor. The new levels also allow for new electronic transitions and hence new absorption peeks will appear in the visual, near infrared and infrared part of the electromagnetic spectrum. The addition of another charge results in a bipolaron, which is two locally coupled polarons. 


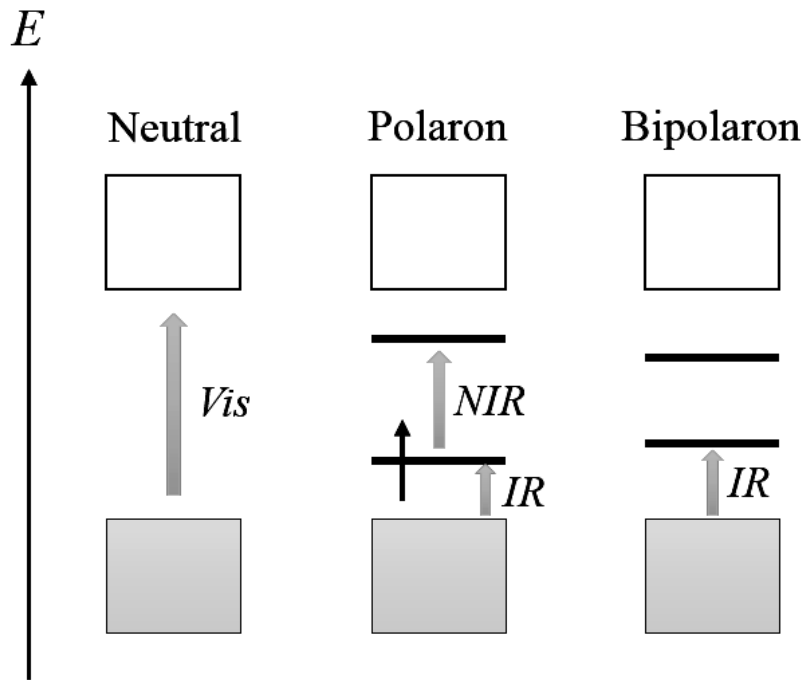

Figure 2.5 New energy levels and optical absorption transitions (grey arrows) in polarons and bipolarons.

Figure 2.5 shows the band diagram of a neutral polymer in comparison to a polymer with polarons or bipolarons. In the neutral polymer, there is only one possible transition of electron excitation due to electromagnetic radiation. The band gap of neutral conjugated polymers typically has a band gap corresponding to visible light (Vis), and they often appear strongly colored to the eye. When an electron is removed from the top of the valence band, a polaron is formed and the singly occupied energy level moves up in energy. At the same time, the lowest lying energy level in the conduction band moves down. This results in two new energy levels, which makes two new electronic transitions possible. The single electron in the polaron band can be excited to the higher polaron energy, resulting in an energy absorption corresponding to radiation in the near infrared (NIR) part of the spectra. An electron can alternatively be excited from the valence band to the polaron band. This transition requires a relatively smaller energy and thus corresponds to radiation in the infrared (IR) region. If an additional electron is removed by oxidation from the polaron band, this band will be empty and the band gap further decreases. These are the bipolaron bands. Since there are no electrons in the bipolaron bands, the only possible excitation (besides the excitation of the neutral polymer) is from the valence band to the bipolaron band. 


\subsubsection{Charge Transport and Disorder}

When an electric field is applied across a conductor, charge carriers move by drift resulting in a net current. In conductive polymers, polarons and bipolarons are the charge carriers and they can move both along a single polymer and jump between different polymers or polymer segments. The limiting factor in transport of polarons is the intermolecular transfer between polymers. The transport depends on several factors such as polaron formation energy, orbital coupling between polymers and energetic and spatial disorder. The polaron formation energy, or more general, the charge reorganization energy $(\lambda)$, is the energy associated with the structural changes of the polymer, hosting the charge, and the surrounding solid when a charge is transferred from one molecule to another ${ }^{[4]}$ In the Marcus theory of charge transfer, the rate $(k)$ of charge transfer is described by Equation 2.1. Here, $T$ is the temperature and $k_{B}$ is the Boltzmann constant. The degree of overlap of the molecular orbitals of two polymers will also effect the charge transfer rate. This overlap is called $\pi-\pi$ stacking, and a large overlap will lead to faster transfer rates.

$$
k \propto e^{-\frac{\lambda}{k_{B} T}}
$$

In an inorganic semiconductor, it is possible to construct almost perfect single crystal structures with few defects. In this type of periodic lattice, charges are delocalized and travel at a characteristic energy level. Polymers on the other hand are most often disordered systems, both with respect to energy and structure. A polymer chain can have chemical or conformational defects which separate the chain into segments of different length. Charges on long segments will have a lower energy than charges on shorts segments. The energy also depends on the stacking of polymers (crystalline or amorphous) and the presence of other charged species or dipoles in the immediate vicinity. This broad range of energy levels is referred to as energetic disorder. In these type of systems, there will be a certain energy known as the mobility edge $\left(E_{c}\right)$ below which charges are localized. The Mobility edge model of transport states that localized charges need to be excited to energies above the mobility edge to take part in charge transport. Conductivity $(\sigma)$ in the Mobility edge model is described by Equation 2.2, where $E_{e q}$ is the equilibrium energy (the most probable energy for charges to occupy). Charges will be remained trapped until they are thermally excited above the mobility edge. 


$$
\begin{aligned}
& \sigma=\sigma_{0} e^{-\frac{E_{C}-E_{e q}}{k_{B} T}} \\
& p \propto e^{-2 \alpha \Delta x} e^{-\frac{\Delta E}{k_{B} T}} \\
& \sigma=\sigma_{0}(T) e^{-\left(\frac{T_{0}}{T}\right)^{\frac{1}{1+d}}}
\end{aligned}
$$

However, charge transport can occur even below the mobility edge by thermally activated hopping and tunneling between localized states. This type of transport known as variable range hopping is the best description of most conductive polymer systems. The probability ( $p$ ) of an excitation to occur depends both on the distance $(\Delta x)$ and energy difference $\left(E_{j}-E_{i}=\Delta E\right)$ between two sites $i$ and $j$. The hopping probability according to the Miller-Abrahams theory of charge transport is described in Equation 2.3 where $\alpha$ is the inverse localization radius. Figure 2.6 shows how a charge at the equilibrium energy can either jump far, to a state with low energy, or make a short jump to high energy. For a given temperature, Equation 2.3 suggests that there should be a tradeoff between jumping high and close or jumping low and far. The energy level to which the probability of a jump from the equilibrium energy is maximized is called the transport level $\left(E_{T}\right)$. Most charges are therefore transported at energies close to the transport level. The Miller-Abraham equations of transport can further be used to derive Mott's variable range hopping model, with the Mott conductivity described by Equation 2.4. In this equation, $T_{0}$ is the characteristic temperature and $d$ is the dimensionality of the material.

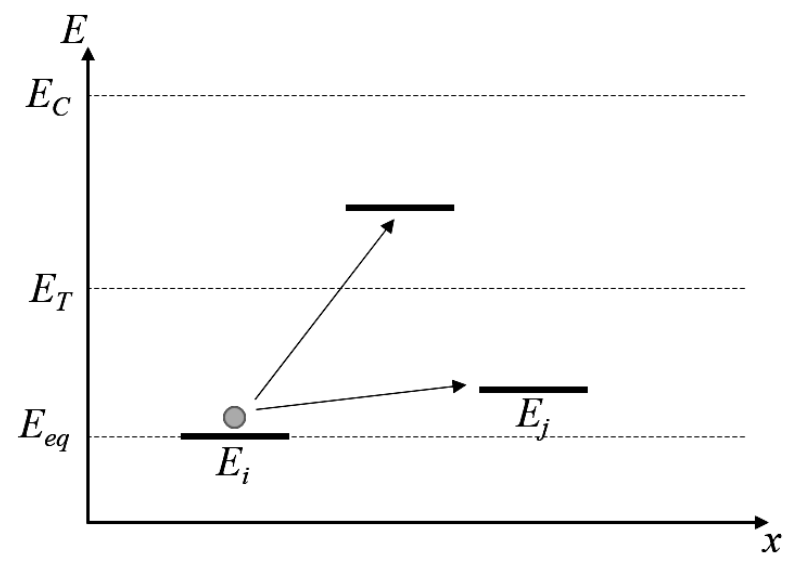

Figure 2.6 Charge transport in variable range hopping. 


\subsubsection{PEDOT}

Poly(3,4-ethylenedioxythiophene), commonly abbreviated to PEDOT (and sometimes PEDT) is one of the most studied and explored conducting polymers. The polymer was developed by Bayer AG research laboratory in the 1980s and became an immediate success due to its chemical stability while in its oxidized state. After the discovery of highly doped trans-polyacetylene, the company initially worked on stabilizing the polymer in its doped state under ambient conditions. Proving difficult to do so, their attention was then turned to thiophenes. The breakthrough came when it was discovered that an oxygenated bicyclic structure greatly improved stability. Like many other organic electronic materials, PEDOT is not water soluble in its intrinsic form. However, by using the polyanion poly(styrene sulfonate) (PSS) as the counterion, stable water emulsions were formed. The PEDOT:PSS complex made solution processing in water possible, which is one of the prime reasons for its popularity in the field of printed electronics. ${ }^{[5]}$

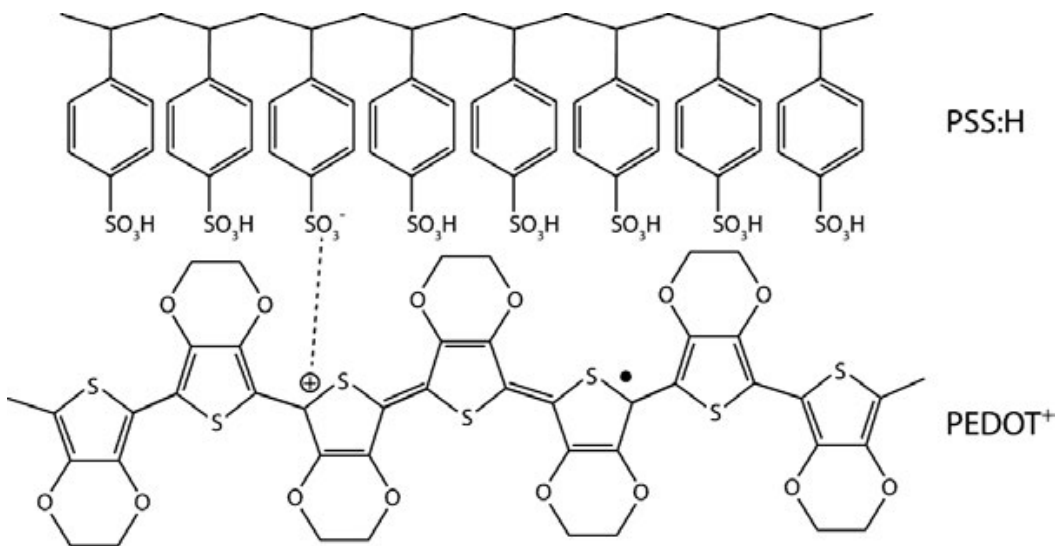

Figure 2.7 The molecular structure of doped PEDOT:PSS with a single polaron.

Figure 2.7 shows the molecular structure of PEDOT:PSS in its singly doped form. An electron has been removed from the polymer resulting in a positive charge. This charge is compensated by the negative charge on one of the sulfonate groups of PSS:H. The positive charge changes the double bond alternation over several repeat units, resulting in a polaron. In its neutral form, PEDOT has a dark blue color and low transparency. Upon oxidation (doping), the color changes to light blue and the transparency increases manyfold due to the shift of the absorption maxima to longer wavelengths. The ability of PEDOT to change color 
upon oxidation and reduction, known as electrochromism (see Chapter 4.4), makes it interesting for applications such as printed displays and smart windows. ${ }^{[6]}$

Although PEDOT:PSS is the most researched form of PEDOT, many other counterions, such as chloride and p-toluenesulfonic acid (tosylate or Tos), have been investigated. ${ }^{[7]}$ The choice of counter ion affects the doping level as well as the packing of the PEDOT chains and thus influences the conductivity. PEDOT:PSS can reach conductivities of $\sim 1000 \mathrm{~S} / \mathrm{cm}$, while values of $2000-5000 \mathrm{~S} / \mathrm{cm}$ have been reported with counterions such as tosylate ${ }^{[8]}$ or trifluoromethanesulfonate[9]. These highly conductive forms of PEDOT have also been shown to, in some cases, transition from semiconducting behavior to semi-metallic behavior. ${ }^{[10]}$
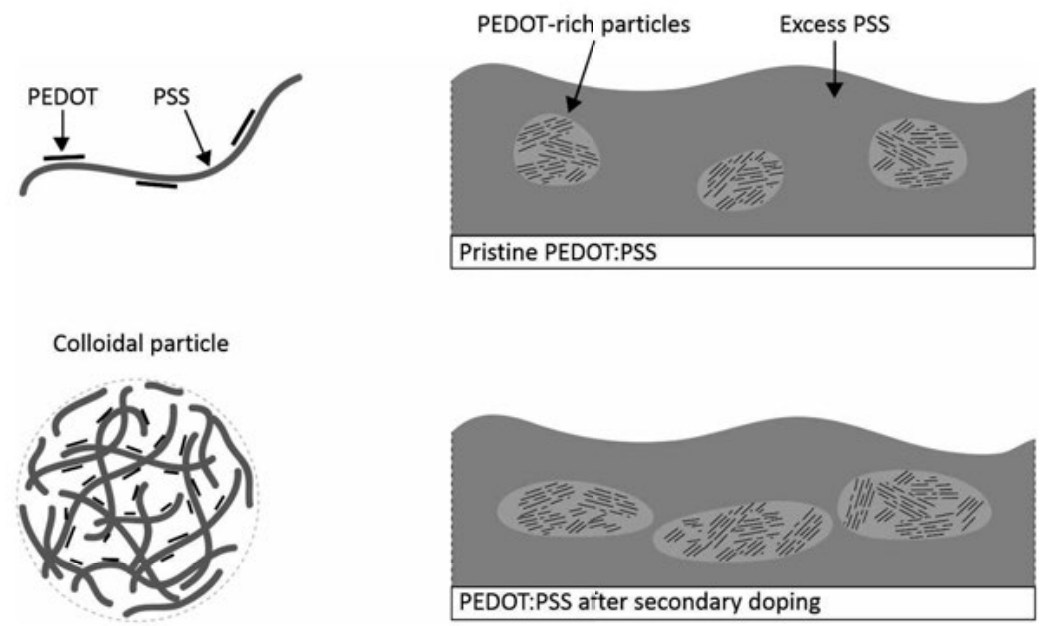

Figure 2.8 Schematic illustration of the structure of PEDOT bonded to PSS, colloidal particles in solution form and the effect of secondary doping in thin films.

Thin polymer films produced from PEDOT:PSS water-emulsions (for example by spin coating) typically have low conductivity $(<0.1 \mathrm{~S} / \mathrm{cm})$. In part, this is due to the excess of PSS, which forms insulating claddings around PEDOT-rich grains. This effect is shown in Figure 2.8. In aqueous solution, PEDOT:PSS forms stable colloidal particles with an outer shell that consists of the water-soluble PSS and a PEDOT rich core. During film formation, this structure will be partly preserved resulting in isolated electronically conducting PEDOT domains with insulating PSS phase around them. The conductivity can be greatly increased by so called secondary doping. The phrase, first coined by MacDiarmid and Epstein, refers to chemicals which can enhance the conductivity of already doped conductive polymers. ${ }^{[11]}$ The 
process often involves adding a high boiling point organic solvent, such as sorbitol or dimethyl sulfoxide, to the PEDOT:PSS emulsion before processing thin films followed by thermal annealing. ${ }^{[12]}$ Alternatively, the polymer film can be immersed in the high boiling point solvent. Although the mechanism of the increase in conductivity is not fully understood, most evidence points to a morphological change. Upon adding a secondary dopant, a modification of the phase separation occurs whereby the PEDOT-rich grains become larger, elongated and better connected by decreasing the thickness of the insulating PSS barrier.[13-16] Since the rate limiting step for charge transport is thought to derive from hopping between the conducting PEDOT-grains, and since the tunneling probability decreases exponentially with distance, even a small change in the barrier thickness can have a great effect on charge transport.

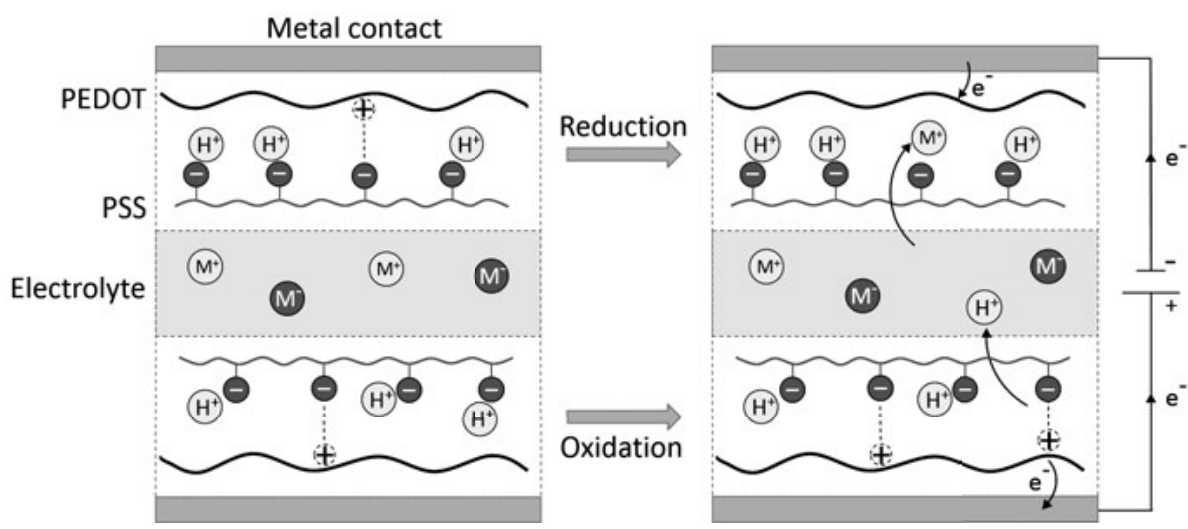

Figure 2.9 Electrochemical reduction/oxidation of PEDOT:PSS.

Originally used as an antistatic coating, PEDOT is now being used in a plethora of different device applications in organic electronics. In organic solar cells, PEDOT is often coated on the transparent conductive electrode to lower the hole-injection barrier. Due to the low thermal conductivity of polymers, it has also been used as the p-type high figure of merit material in thermoelectric generators (Chapter 4.3). The ability of PEDOT to change doping state upon electrochemical reduction and oxidation has resulted in applications such as organic electrochemical transistors (Chapter 4.2), supercapacitors (Chapter 4.1.3) and electrochromic devices (Chapter 4.4). Figure 2.9 illustrates the fundamental ion exchange and charge accumulation mechanisms, which govern the mode of operation in these devices. Two metal electrodes coated with PEDOT:PSS are connected by an electrolyte in an electrochemical cell. The electrolyte contains mobile anions ( $\left.\mathrm{M}^{-}\right)$and cations 
$\left(\mathrm{M}^{+}\right)$. When applying a voltage bias between the electrodes, the PEDOT on the side connected to the negative terminal becomes reduced while the PEDOT connected to the positive terminal gets (further) oxidized. As PEDOT becomes reduced, the negatively charged sulfonate groups that were balancing the positive charges on PEDOT are left uncompensated. Cations from the electrolyte migrate into the polymer film to compensate the uncompensated PSS charge to maintain overall neutrality. On the other side of the cell, new positive charges are added to the PEDOT chains. These charges are compensated by the previously protonated sulfonate groups or by anions from the electrolyte. The reduction-reaction is described by Equation 2.5. The ion exchange mechanism will be different in systems where the counterion to PEDOT is not a large immobile molecule. Also, even for PEDOT:PSS, when put into an electrolyte solution, some of the mobile ions will move into the polymer film and take over the role as the charge compensating ions, making the complete picture of ion flux during electrochemical switching more complicated than the above described reaction.

$$
\mathrm{PEDOT}^{+}: \mathrm{PSS}^{-}+\mathrm{M}^{+}+e^{-} \rightarrow \mathrm{PEDOT}^{0}+\mathrm{PSS}^{-}: \mathrm{M}^{+}
$$

The depletion of the positive charge carriers on the reduced side results in a dramatic drop in conductivity along with a change in color from transparent to dark blue. The difference in the oxidation state, and thereby the electrochemical potential, of the two electrodes is the basis for using PEDOT as the active material in supercapacitors.

\subsection{Cellulose}

\subsubsection{Wood Fiber Structure}

Cellulose is the most abundant biopolymer on the planet and can be found in the cell walls of plants as well as in certain algae and bacteria.[17] Even some (simple) animals, like tunicates, can produce cellulose. The cellulose molecule consists of several hundreds or thousands of D-glucose units (Figure 2.10) and is the main component in the production of paper. The main source of cellulose used in industry is wood which is processed into pulp by a combination of mechanical and chemical treatments to separate the wood fibers. The cell walls of plants consists of bundles of fibers with a diameter of 20-50 $\mu \mathrm{m}$ which in turn consist of smaller nanofibrils with a diameter of 5-10 nm. ${ }^{[18]}$ The nanofibrils further consist of elementary fibrils with a diameter of 1.5-3.5 nm (Figure 2.10). The wood fibers 
also contain lignin and hemicellulose which fill the space between the fibers and fibrils and bind them together. The chemical and mechanical treatment in pulp production separates the fibers and removes lignin and hemicellulose. Different types of pulp contain different amounts of lignin and hemicellulose depending on the type of treatment. So-called kraft pulp consists mainly of cellulose which result in strong paper.

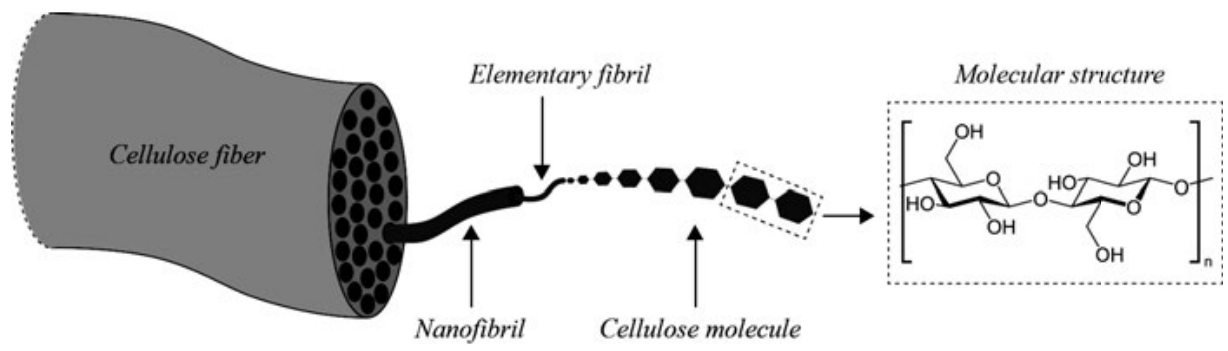

Figure 2.10 The macro, micro and nanostructure of wood fibers.

\subsubsection{Nanocellulose}

The term nanocellulose refers to several cellulose based materials where the smallest particle dimension is 1-100 $\mathrm{nm}$. The three main types of nanocellulose are: nanocrystalline cellulose (NCC) or cellulose nanocrystals (CNC), nanofibrillated cellulose (NFC) or cellulose nanofibrils (CNF), and bacterial nanocellulose (BNC).[19] Other names include microcrystalline cellulose (MCC) or microfibrillated cellulose (MFC), and there is often confusion about whether the terms are synonymous with NCC and NFC. In a proposed new TAPPI standard, the terms were classified according to Figure 2.11.[20]

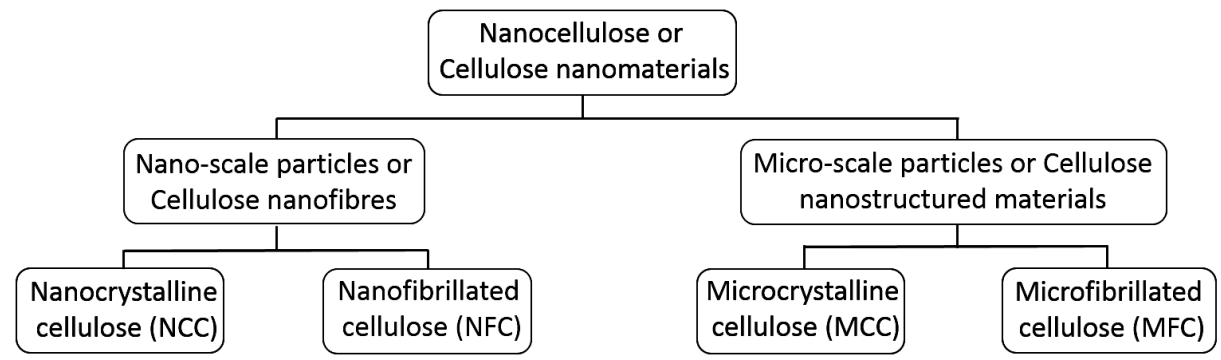

Figure 2.11 Terminology of wood-based nanocellulose adapted from TAPPI standard WI 3021.[20]

NFC consists of cellulose fibrils with diameter of 2-10 $\mathrm{nm}$ and length of $>10$ $\mu \mathrm{m}$. These fibrils consist of both crystalline and amorphous regions of cellulose 
which makes them both strong and flexible. By acid hydrolysis, the amorphous regions can be dissolved resulting in highly crystalline NCC. NCC have similar diameters to NFC but are considerably shorter (100-600 nm) and since NCC has a higher crystallinity, they are more rigid compared to NFC. While NFC and NCC are often produced from plant lignocellulose, bacterial cellulose (sometimes referred to as microbial cellulose) is derived from certain kinds of aerobic bacteria, such as acetic acid bacteria. Since these bacteria don't have any lignin or hemicellulose, the BNC have a high purity and crystallinity.

There is a big interest in both NFC and NCC due to their mechanical strength, barrier properties and optical properties. With a tensile strength comparable to steel $(\sim 200 \mathrm{MPa})$, they have been proposed as a strengthening additive in many materials. ${ }^{[21]}$ Paper produced from NFC, so-called nanopaper, can be made very smooth and has therefore been explored as a substrate for flexible and printed electronics. ${ }^{[22]}$ Due to the small fibril dimensions, these papers also typically have a high transparency. Furthermore, the strength and high specific surface area of NFC has prompted its use in various paper-electronics applications (see Chapter 3.3).

\subsubsection{Nanofibrillated Cellulose}

NFC can be produced from several cellulose sources by mechanical processes such as high-pressure homogenization ${ }^{[23]}$, microfluidization ${ }^{[24]}$ or high-intensity ultrasonication ${ }^{[25]}$ whereby the nanofibrils are liberated from the cellulose fibers. This process requires large amounts of energy in the order of 30,000 kWh/tonne, which has hindered large scale production of NCF for use in commercial applications. ${ }^{[26]}$ The energy consumption can be dramatically reduced by different types of chemical or enzymatic treatments prior to the mechanical treatment. These treatments include TEMPO-mediated oxidation, enzymatic hydrolysis and carboxymethylation. Isogai et al. used TEMPO-mediated oxidation and demonstrated an energy reduction of $95 \%{ }^{[27]}$ The treatment involves the functionalization of the glucose units to form carboxylic groups. The negative charge on the carboxylic groups results in electrostatic repulsion between the fibrils which facilitate their separation. The repulsion between the charged fibrils also aid in forming stable colloidal suspensions of NFC in water.

NFC-based materials have been fabricated with different form factors. This includes films (nanopaper) ${ }^{[21]}$, filaments ${ }^{[28]}$ and aerogels/foams ${ }^{[29]}$. Various applications of nanopaper in electronics as well as the use of NFC as a component 
in composite electroactive materials is covered in Chapter 3.3. Håkansson et al. developed a method to produce very strong NFC filaments or threads by aligning cellulose fibrils in a water suspension by pushing it through a narrow channel while adding a salt solution. ${ }^{[28]}$ The ions from the salt screen the charge from the carboxylic groups in the cellulose fibrils and reduce their electrostatic repulsion which causes the cellulose suspension to form a hydrogel. The filaments could potentially be used as an alternative to silk in the production of fabrics. Aerogels are 3D polymer networks with high porosity and low density. Aerogels based on cellulose and NFC have been used for applications such as thermal insulation ${ }^{[30]}$, biomedical applications ${ }^{[31]}$ and energy storage ${ }^{[32]}$.

\subsection{Quinones}

With the expansion of renewable energy production, there is a growing need for equally green energy storage devices. To this end, there is a growing interest in batteries, supercapacitors and hybrid systems based on organic materials such as biopolymers. Quinones are a class of organic compounds which are derived from the oxidation of certain aromatic molecules. Quinones of different types can be found in plants and animals, but they can also be produced synthetically. In plants, they take part in the charge transfer mechanism in photosynthesis and in animals they are found in certain vitamins and play an important role in the respiratory system. Many organic dyes, both natural and synthetic, are quinone derivatives. One such example is alizarin (1,2-dihydroxyanthraquinone) which is a red dye that has been used in the coloring of fabrics for several thousand years.

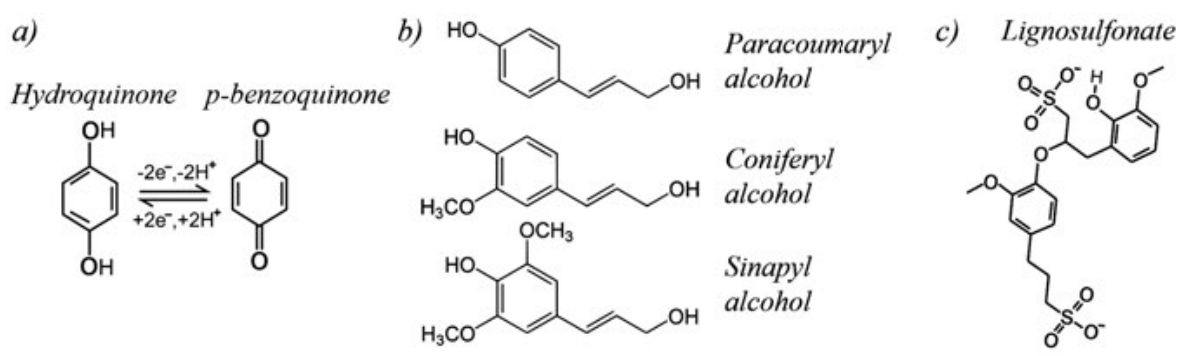

Figure 2.12 (a) Hydroquinone/benzoquinone redox reaction, (b) the monolignols of lignin and (c) lignosulfonate.

Quinones have a conjugated structure and can be reduced into hydroquinone by taking up two electrons and two protons. Figure 2.12a shows the redox reaction of hydroquinone and para-benzoquinone. The reversible redox activity of 20 
quinones makes them ideal for use in organic redox batteries and similar charge storage devices. With a theoretical charge storage capacity of $496 \mathrm{mAh} / \mathrm{g}$, quinones have the potential to rival even certain lithium ion batteries.[33] Quinones can be dissolved in the electrolyte of a supercapacitor ${ }^{[34]}$ or a flow battery[35], or they can be incorporated into the electrodes of the energy storage devices. The quinone molecules must be added to a conductive matrix to transfer the electrical charge in the redox reaction. Carbon and conductive polymers are frequently used as the conductive material in hybrid supercapacitor/battery systems. Such composite materials have been synthesized by chemically bonding the quinones to carbon surfaces $^{[36]}$ or conductive polymers ${ }^{[37-38]}$. Another strategy is to synthesize conductive polymers in the presence of quinones to form an interpenetrating conductive network. [33, 39-40]

Lignin is a biopolymer which can be found in the cell walls of plants. It is the second most abundant biomolecule after cellulose. Lignin is a complex polymer which is mainly composed of three monolignols: Paracoumaryl alcohol, coniferyl alcohol and sinapyl alcohol (Figure 2.12b). The phenolic groups in these alcohols are cheap, abundant and environmentally friendly sources of quinones. Sulfonated lignin, or lignosulfonates (LS), are a byproduct from the production of paper pulp when sulfite pulping is used. The large lignin polymer is broken apart and sulfonate groups are attached to the fragments which makes the molecules water soluble. Figure 2.12c shows one possible molecular structure of LS, although they come in a large variety of molecular weight. LS has been used as the source of quinones in many capacitor and battery applications where they are formed from the phenolic groups of LS by oxidation. ${ }^{[33,39-44]}$

\subsection{Electrolytes}

An electrolyte is a substance which dissociates into free ions when dissolved in a polar solvent and which can move through the solvent under the influence of an electric field, thus constituting an ionic conductor. The electrolyte can be a salt, acid or base dissolved in a liquid or solid solvent. Electrolytes are important in electrochemistry and electrochemical devices since an electrochemical cell always contains at least two electrodes separated by at least one electrolyte phase. There are several different types of electrolytes which are used for different electrochemical applications. Some of these electrolytes are depicted schematically in Figure 2.13. 
In liquid electrolytes, the solvent can be either aqueous or organic. There are also solvent free liquid electrolytes, so called ionic liquids, which are molten salts with a melting point below room temperature. Liquid electrolytes generally have higher ionic conductivity than solid electrolytes. Among solid electrolytes there are polymer electrolytes, gel electrolytes, polyelectrolytes as well as certain inorganic solids which can conduct small ions like $\mathrm{H}^{+}$and $\mathrm{Li}^{+}$. One of the advantage of solid electrolytes is that leakage from electrochemical devices is minimized, which is otherwise a common cause of device failure. Also, some solid electrolytes can be processed more easily compared with liquid electrolytes during device fabrication. For example, polyelectrolytes have been used in printed electronics in the fabrication of electrochemical devices. ${ }^{[45-46]}$ A polymer electrolyte is a polymer blended with a salt. Poly(ethylene oxide) (PEO) has been used as a solid electrolyte in lithium ion batteries by blending it with different lithium salts.[47] A gel electrolyte is a liquid electrolyte blended with a polymer to obtain a semi-solid state. Polyelectrolytes are polymers with electrolytic groups ionically bonded to smaller counterions. One such example is poly(styrene sulfonate) (PSS) which is a polyanion with negative sulfonate groups. Similar to ionic liquids, polyelectrolytes don't require any additional solvent. However, the ion mobility in a polyelectrolyte is highly dependent on the amount of absorbed atmospheric water and is therefore sensitive to relative humidity.

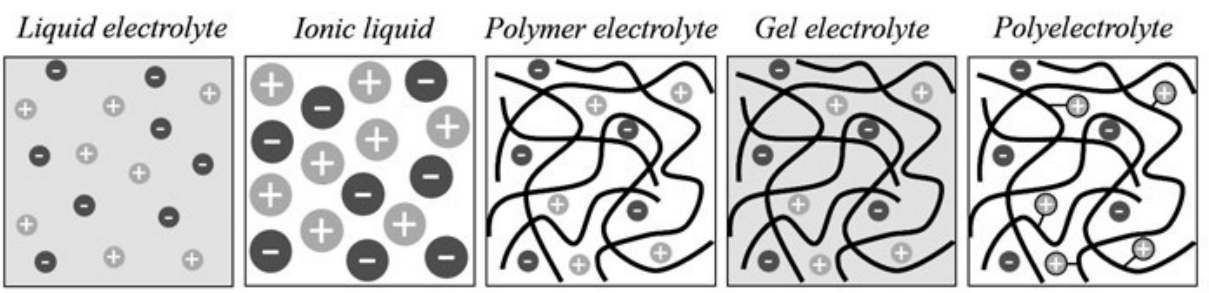

Liquid solvent

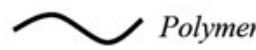

- Anion

Cation

Figure 2.13 Different types of liquid and solid electrolytes.

The performance of electrochemical devices depends largely on the properties of the electrolyte being used. In a supercapacitor, two porous electrodes are separated by an electrolyte (see Chapter 4.1.3). This type of device is used to store energy in the form of electrical charge at the electrode/electrolyte interface. The capacitance of such a device is often attributed to the electrodes, and is often 
expressed as $\mathrm{F} / \mathrm{g}$ or $\mathrm{F} / \mathrm{cm}^{2}$ where the mass and area is that of the electrode material. However, the capacitance also depends on the choice of electrolyte. The capacitance is proportional to the dielectric constant of the solvent and inversely proportional to the thickness of the double layer. The double layer is determined by the Debye length which in turn is dependent on both the dielectric constant of the solvent and the ion concentration in the electrolyte.[48] Furthermore, the size of the ions, the solvent molecules and the solvation shell around the ions affect the permeability of the ions inside the porous electrodes and thereby effect the capacitance. [49]

The maximum power at which a capacitor can be charged and discharged depends on the equivalent series resistance of the device. One component of this resistance is the electrolyte resistance. This resistance is inversely proportional to the concentration of ions and the mobility of the ions in the solvent. The maximum energy which can be stored in a capacitor is proportional to the capacitance and voltage squared. The voltage is limited by the electrochemical stability of the electrolyte. In aqueous electrolytes, the voltage is limited to $1.23 \mathrm{~V}$ by the electrolysis of water. Organic solvents are often used because of their better electrochemical stability. However, the ion concentrations which can be achieved in organic solvents is often lower than in water, which increases the electrolyte resistance and thus lower the power.

Ionic liquids have superior electrochemical stability compared to both aqueous and organic electrolytes and can withstand 4-10 V.[50-51] Furthermore, they exhibit good ionic conductivity ${ }^{[52]}$ and because they have no hydration shell, high values of capacitance can be achieved in nanoporous electrodes. ${ }^{[53]}$ The limiting factor for using ionic liquids in various commercial device applications is their relatively high price. However, with new processing methods, this cost can be reduced to a point where it is comparable with organic solvents such as acetone. ${ }^{[54]}$ 


\section{Flexible Organic Electronics}

\subsection{Printed Electronics}

Printed electronics is the usage of common printing methods to manufacture devices by using inks with electronic or electrochemical functionality. Some of the printing techniques used in this field are screen printing, inkjet printing, flexographic printing and gravure printing. Printed electronics has several advantages over conventional electronics manufacturing such as low cost, high throughput, large area fabrication and the possibility to manufacture electronics on cheap and flexible substrates such as paper or plastics. The drawbacks with this manufacturing method include the limited patterning resolution and generally lower performance of the produced devices compared to those manufactured with conventional methods.

For a material to be used in printed electronics, it must be possible to process it in the form of an ink. Organic materials are generally easy to process in solution form, but also inorganic materials such as metals can be added to an ink, for example in the form of particles suspended in a polymer matrix. Conductive polymers, polyelectrolytes and small organic molecules with electronic functionality are ideal for usage in printing processes. As such, many organic electronics devices have been realized in this way, including thin film transistors [55], electrochemical transistors [56], electrochromic displays[46], organic light emitting diodes ${ }^{[57-59]}$ and organic solar cells ${ }^{[60-61]}$. Conductive inks of carbon and metals are commercially available, but since the inks generally contain particles of these materials, the resulting printed layers will have higher resistivity than their bulk counterparts due to poor connection between the particles.

\subsection{Photopatterning of Conductive Polymers}

Although printed electronics hold great promise for the fabrication of organic electronic components, due to the limited resolution of most printing techniques (10-100 $\mu \mathrm{m})$, other techniques are sometimes needed to pattern materials such as conductive polymers. Some techniques such as mold transfer printing [62] and dip pen nanolithography ${ }^{[63]}$ have been used to pattern organic semiconductors with sub-micrometer resolution. However, the most commonly used method for high resolution patterning of both organic and inorganic materials is photolithography, 
which involves the patterning of a light sensitive polymer (photoresist) using UVlight. Figure 3.1 shows the principle behind the technique. The photoresist is coated on a substrate ( $i$ ) after which it is irradiated with UV-light through a photomask (ii). The photomask can be made from quartz glass (which is transparent to UV-light) with a metal pattern which will block the passage of the UV-light in certain areas. The UV-light will either crosslink (positive photoresist) or break down (negative photoresist) the photoresist depending on what type of resist is used, and after further chemical treatment with a developer, either the areas that were irradiated or not irradiated will be removed (iii).
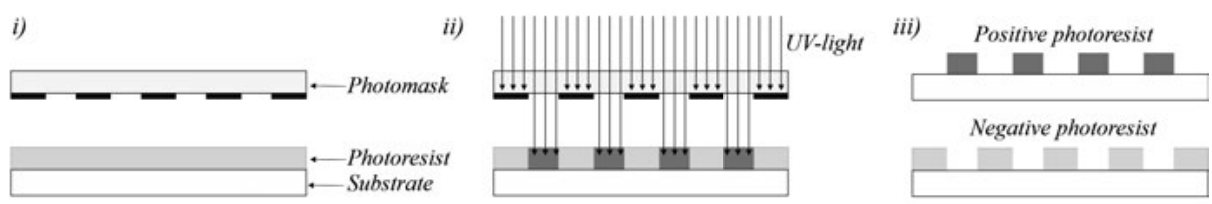

Figure 3.1 Schematic of the photolithography procedure.

The patterned photoresist can be used as a mold for patterning conductive polymers, either by adding them in solution form or by chemical or electrochemical polymerization. Alternatively, the photoresist can be added on top of a layer of the conductive polymer after which the photoresist pattern is used as a mask for etching. In this way, high resolution organic devices, such as organic field effect transistors, can be fabricated. However, there are several drawbacks with using photolithography for patterning organic electronic devices: (1) Photolithography involves many processing steps of baking, spinning, exposure developing and stripping and is therefore time consuming. (2) Conductive polymers are often sensitive to the chemicals used to develop and strip the photoresist.[64] (3) Wet etching is a common processing step in photolithography which is both complicated and uses harsh chemicals. (4) The photoresist must be compatible with the solvent used to disperse the conductive polymer. (5) The complicated processing steps and hazardous chemicals used in photolithography makes it unsuited for large area fabrication of devices.

To overcome the above-mentioned issues, new strategies to reduce the number of processing steps ${ }^{[65]}$, develop less harmful solvents ${ }^{[66]}$ and new innovative processing steps ${ }^{[64]}$ have been explored. In addition to this, direct patterning techniques of conductive polymers such as PEDOT using UV-light, but without the usage of a photoresist have been developed.[67] These techniques 
involve the selective modulation of the conductivity in conductive polymer films and are not dependent on a photoresist.

\subsection{Paper Electronics}

Paper is produced by pressing wet cellulose fibers to form thin membranes. The manufacturing of paper can be traced back as far back as the $2^{\text {nd }}$ century and has had a large impact on the course of history due to the preservation and spreading of information by writing. Although paper is still widely used in our society in the form of books, newspapers and household products, the invention of the computer and the internet has opened a new and faster way of transferring information which has led to the decline in the usage of paper. However, paper is gaining renewed interest in the form of paper electronics.

Paper electronics encompasses applications where paper is either used as a flexible carrier substrate for electronics ${ }^{[68]}$ or as an integrated part of the electronic components ${ }^{[69]}$. Printed electronics usually use either paper or plastic as the substrate. When electronics are printed on flexible plastic sheets this is sometimes referred to as paper-like electronics. Recently there has been a growing interest in using paper as more than a carrier substrate for 2D-electronics and to integrate electronics into the bulk of the paper. There are several reasons to use paper/cellulose as a scaffold or binder for composite materials in electronics: (i) cellulose is environmentally friendly, cheap, abundant and combustible, (ii) it is flexible and strong (iii) the intrinsic large specific surface area of paper is ideal for a number of applications such as supercapacitors and batteries to increase the active area, (iv) the porosity of paper makes ion penetration easy which is important for many electrochemical devices, $(v)$ the biocompatibility of cellulose opens up for applications such as biofuel cells.

Conductive paper: Flexible electronic devices typically require conductive electrodes to supply and collect charge. If these conductors are coated on top of a substrate there is usually a limit to how thick they can be made without cracking during bending. By functionalizing paper with conductive materials such as carbon nanotubes (CNT) or conductive polymers, the full bulk of the sheet can be utilized to lower the sheet resistance. Another added advantage is the possibility of passing a current through the bulk of the paper which makes contacting on both sides possible.

The two most commonly reported conductive materials used to functionalize paper are carbon and conductive polymers. These materials exhibit a good 
interaction with the cellulose fibers/fibrils and can form a uniform coating on the fibers. ${ }^{[70]}$ Carbon, in the form or graphite, CNT or graphene, has been used both for coating and functionalizing paper. When making a composite material by blending a conductor with an insulating polymer, it is important to get a homogeneous blend to form a percolating conductive network. Carbon can either be added to paper by soaking it in a functionalized ink or by mixing it with the cellulose water dispersion during paper manufacturing. In both cases, it is necessary to disperse the carbon in a liquid solvent (typically water-based to have good interaction with paper). Both graphite and CNT are difficult to disperse in water solutions without the use of surfactants, which often limit the conductivity by forming insulating layers between the conductive particles. Nanofibrillated cellulose (NFC) water suspensions have been shown to have excellent colloidal stabilizing properties for both graphite particles and CNT, making it possible to produce high quality conductive papers.[71-72]

Conductive polymers can be composited with paper either by adding it in the form of a liquid suspension or by direct polymerization onto the cellulose fibers in solution or on paper.[73-76] It has been demonstrated that the type of cellulose and the surface charge of the fibers have a strong influence on the quality of the polymer coating on paper and hence the conductivity. Qian et al. investigated the influence of the pulp type on the polymerization of polyaniline (PANI) on cellulose fibers. ${ }^{[76]}$ They observed that chemical pulps with charged groups on the cellulose fibers resulted in thicker layers of PANI compared to high yield pulps. They could also see a linear relationship between the sheet resistance and the amount of charged groups on the fibers with sheet resistance decreasing with increasing surface charge. Sasso et al. polymerized polypyrrole (PPy) in different mixtures of carboxymethyl cellulose (CMC), xylan (hemicellulose) and NFC to manufacture conductive nanocomposite films. ${ }^{[73]}$ They observed that the films containing NFC had larger PPy particles which formed networks between the fibrils. Nanocomposites with CMC had two orders of magnitude lower conductivity compared to NFC which was attributed to insulating phases of CMC forming around the PPy particles.

Inorganic materials have also been used to prepare conductive or semiconducting paper for various applications. Sani et al. fabricated selfsupporting semiconducting films of silicon microparticles and NFC which were used to make high frequency diodes. ${ }^{[77]}$ Sandberg et al. incorporated zinc oxide tetrapods in paper pulp to make photoconductive paper. This paper was fabricated in a pilot scale paper machine at a rate of 100 meters per minute. After adding 
screen printed carbon electrodes, the paper could be used as a sensor for UV-light. Lastly, Nogi et al. incorporated silver nanowires in NFC to make highly transparent and conductive paper. This paper was further used as the transparent electrode in a flexible solar cell with performance as high as for ITO solar cells. ${ }^{[78]}$

Energy storage: Electrochemical energy storage devices such as supercapacitors and batteries typically consist of two electrodes separated by an ion-conductive layer (electrolyte). The electrodes host the active material while the electrolyte supply and/or conduct charge compensating ions. To achieve good device performance, it is important that both ions and electrons can access the active material in an optimal way. This has led to the development of conductive electrodes with high specific surface area and porosity onto which active materials can be coated. The high specific surface area ensure that a large amount of the active material is exposed to the electrolyte and high porosity enables ion transport into the bulk of the electrode. This makes paper, with its intrinsic porous structure, an interesting candidate for building charge storage devices with the added advantage of flexibility. ${ }^{[69]}$

Paper-based supercapacitors have been constructed by functionalizing cellulose with carbon[72], conductive polymers ${ }^{[74,79]}$ or metal oxides ${ }^{[80]}$. Both Gui et al.[81] and Chen et al.[80] investigated the role of mesoporosity in cellulose on the performance of electrochemical energy storage. They concluded that not only the spaces between the cellulose fibers, but also the porous structure of the fibers themselves, contribute to enhanced ion transport by creating electrolyte reservoirs and ion pathways. Andres et al. demonstrated how the introduction of NFC in graphite-based supercapacitors not only enhanced the capacitance of the devices but also improved their lifetime by acting as a mechanically supporting matrix which prevented cracking of the graphite during cycling measurements. ${ }^{[72]}$

There are also many reports of paper-based batteries which have the same advantages as supercapacitors. ${ }^{[82-83]}$ The flexibility of such paper devices has resulted in a completely new battery device concept: origami batteries. By folding a flexible paper-battery in a Miura pattern, Cheng et al. was able to improve the areal energy density of their device.[84]

Energy harvesting: Flexible electronics can potentially be used in applications such as wearable electronics where strain on the devices arises from the movement of the body. To be of practical use, such systems need to be selfpowered, but at the same time lightweight. Although flexible batteries exist, this would mean an extra weight and require periodic recharging. The best scenario would be if the devices could harvest enough energy from their environment to 
operate indefinitely. Several such power-generating devices have already been realized in the form of paper-electronics.

$\mathrm{ZnO}$ is a piezoelectric material which can be used to harvest electrical energy from mechanical energy. By growing $\mathrm{ZnO}$ in the form of nanorods, the bending of these rods will induce a potential difference which can be used to drive an electrical current. Nguyen et al. prepared $\mathrm{ZnO}$ nanorods on paper substrates and demonstrated how mechanical stimulation of the device resulted in a power output.[85] This type of device is ideal for wearable electronics since it can harvest energy directly from the movement of the body. Similar to piezoelectric generators, flexible and paper-based triboelectric generators are another type of device which can harvest energy from movement. [86]

Beside mechanical energy, heat and light are also possible sources for powering flexible electronics.[87] Thermoelectric generators can convert the thermal energy in a temperature gradient into electrical energy. This could be the temperature difference between the body and the surrounding air in a wearable device. Furthermore, flexible organic solar cells with functionalized transparent paper electrodes could be used to harvest light energy.[88]

Microfluidics and sensors: Paper has been used for sensing applications for a long time due to the high porosity and the hygroscopic nature of cellulose. This allows for easy absorption of liquids such as blood or water samples. One of the simplest and oldest examples of a paper sensor in the litmus paper $\mathrm{pH}$ indicator. A filter paper is soaked with a mixture of $\mathrm{pH}$ sensitive dyes (litmus) which change color depending on the $\mathrm{pH}$. There is a large interest in developing paper-based diagnostics systems for disposable self-tests and point-of-care diagnostics for doctors or scientists working in areas where large diagnostic tools cannot be brought. [89-91] These systems are sometimes called micro total analysis systems ( $\mu$ TAS).

Many existing portable diagnostic tools use microfluidics to transport precise amounts of liquids to the sensors, but are generally bulky and rigid. To realize flexible paper-based $\mu$ TAS, both the sensors, electronics and microfluidics must be integrated into the paper. The fabrication of such devices usually start with patterning a porous paper with a hydrophobic material like wax, PDMS or photoresist to define hydrophobic and hydrophilic regions and pathways. Such patterning has been demonstrated using wax printers ${ }^{[2]}$, inkjet printers ${ }^{[93]}$ and photolithography ${ }^{[94]}$. The uncoated parts of the paper can be used as microfluidic channels to wick fluids, but they can also be used to selectively functionalize the paper by adding electronic materials in liquid forms. Hamedi et al. demonstrated 
how wax printing could be used to pattern conductive pathways in paper by adding liquids containing conductive polymers and carbon nanotubes. ${ }^{[95]}$ Such patterning strategies could further be used to realize electronic circuits and sensors inside paper. 


\section{Devices}

\subsection{Capacitors}

Capacitors are devices used to store and release electrical energy and they are one of the most fundamental components in analog and digital electronics. Capacitors have superior power density compared to other energy storage devices, such as batteries, but are often lacking in energy density. Capacitors and batteries are therefore often used together due to their complementing properties.

\subsubsection{Capacitance}

Capacitance is the ability of an object to store energy in the form of electrical charge. When two conductors have different electrical potentials, an electric field exist between them which strength depends on their separation. The charges in the two conductors have different potential energy, and so energy is stored in the system. If the conductors were to be put in electrical contact, charges would move from one side to the other until the potential is equalized. Vice versa, if two conductors at equal potentials are connected by an external voltage, charges will flow from one side to the other until the potential difference equals that of the source. The amount of charge $(Q)$ which can be stored when a certain voltage $(V)$ is applied depends on the geometry of the objects as well as the separation and medium between them. Capacitance $(C)$ has the SI unit Farad (F) and is defined as the ratio between the stored charge and the potential difference (Equation 4.1). The energy $(W)$ which can be stored in a capacitor is determined by its capacitance and the applied voltage according to Equation 4.2.

$$
\begin{aligned}
& C=\frac{Q}{V}=\frac{\varepsilon A}{d}=\frac{\varepsilon_{0} \varepsilon_{r} A}{d} \\
& d W=V d q=\frac{q}{C} d q \rightarrow W=\frac{1}{C} \int_{0}^{Q} q d q=\frac{Q^{2}}{2 C}=\frac{C V^{2}}{2}
\end{aligned}
$$

The most common geometry of capacitors is shown in Figure 4.1a and consists of two parallel conductive plates of area $A$, separated by a distance $d$. The parameter $\varepsilon$ in Equation 4.1 is the permittivity and describes how well electric fields will propagate through the medium (dielectric) between the plates. $\varepsilon_{0}$ is the 
permittivity of free space and $\varepsilon_{r}$ is the ratio between the permittivity of the medium and $\varepsilon_{0}$. The latter is also known as the relative permittivity or the dielectric constant of a material.

As the capacitor is being charged, positive charges accumulate on one plate and negative charges on the other. These charges will repel each other and counteract further charge accumulation. However, if the two plates are brought close together, the charges on one plate will feel the attraction from the opposite charges on the other plate, making it possible to inject more charges. Therefore, the capacitance is inversely proportional to the distance between the plates.

The charge on the two plates induces an electric field $(E)$ across the capacitor. Inside a dielectric material there are randomly aligned electric dipoles (Figure 4.1b) which will be aligned by the electric field (Figure 4.1c). The dipoles now generate their own electric field in the opposite direction of the applied field which lowers the potential difference between the plates allowing more charge to be accumulated. Alternatively, dipoles can be induced in dielectrics which do not possess permanent dipoles. A material with a high dielectric constant will have a large degree of polarization which will lower the electric field and therefore increase the capacitance.

a)

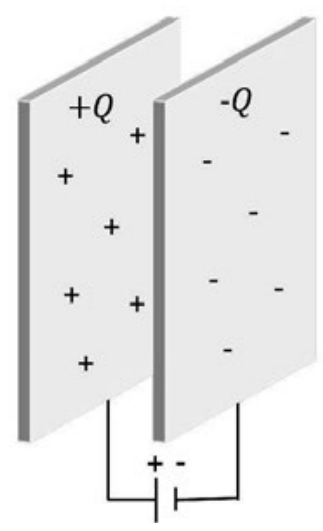

b)

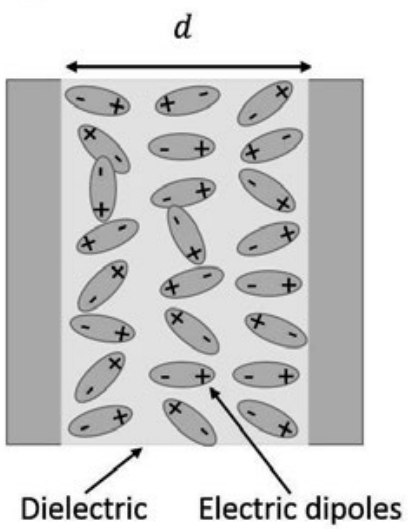

c)

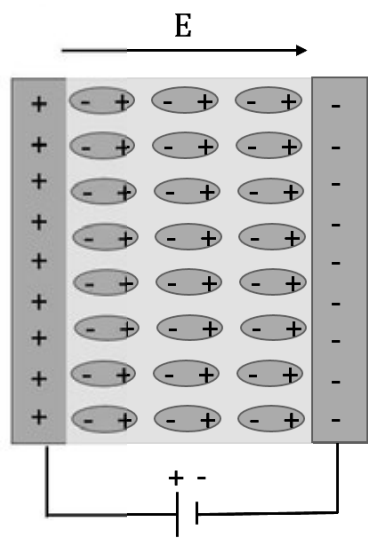

Figure 4.1 Schematic of a parallel plate capacitor (a), cross section without applied voltage (b) and cross section with applied voltage (c).

Two common types of capacitors are film capacitors and ceramic capacitors. Film capacitors use a polymer layer (e.g. paper or plastic) as the dielectric layer 
while ceramic capacitors use a ceramic material. Typical values of commercial film and ceramic capacitors used in electronics range from $1 \mathrm{pF}$ to $1 \mathrm{mF}$.

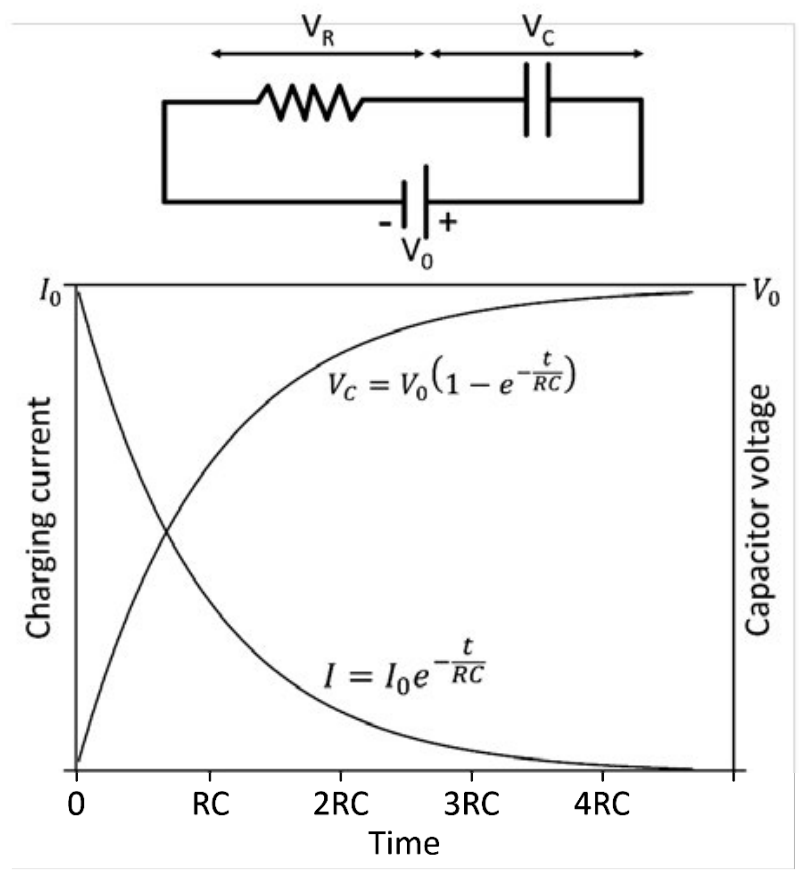

Figure 4.2 The charging behavior (bottom) of an RC circuit (top).

An ideal capacitor can be charged instantaneously and have no limit to how much charge can be stored with increasing voltage. A physical capacitor on the other hand has a characteristic charging time, and the maximum amount of charge that can be stored is limited by the breakdown voltage of the dielectric. Such a capacitor can be modeled as an ideal capacitor with capacitance $C$ in series with an ideal resistor with resistance $\mathrm{R}$. This is known as an RC circuit and is illustrated in Figure 4.2. When a voltage $V_{0}$ is applied over the circuit, a current will run until the capacitor is fully charged. Figure 4.2 also show the current $(I)$ and the voltage across the capacitor $\left(V_{c}\right)$ during charging. The relationship between the applied voltage, the voltage over the capacitor and the voltage over the resistor $\left(V_{R}\right)$ is described by Equation 4.3. At $\mathrm{t}=0$, the current is at its maximum and equal to $V_{0} / R$. At this point, there is no voltage across the capacitor. As the capacitor charges, the current goes down as the voltage across the capacitor increases. When the capacitor is fully charged, $\mathrm{V}_{\mathrm{c}}=\mathrm{V}_{0}$ and no current is running through the circuit. The 
time axis on the graph in Figure 4.2 is in units of $\mathrm{RC}$, which is also known as the time constant, or $\tau$. At $t=3 R C$ the capacitor is charged to $95 \%$.

$$
V_{0}=V_{R}+V_{C}=I(t) R+V_{0}\left(1-e^{-\frac{t}{R C}}\right)
$$

\subsubsection{The Electric Double Layer}

If the dielectric material between the metal plates described in Chapter 4.1.1 is replaced with an ionic conductor, i.e. an electrolyte, the mobile ions will be attracted to the charged plates (Figure 4.3a-b). This type of capacitor is called an electric double layer capacitor since a layer of ions will be built up on each capacitor electrode (plate). Most of the electric field will be concentrated at the interface between the metal and the electrolyte while the potential drop in the bulk of the electrolyte will be small. The capacitance of electric double layer capacitors is therefore less dependent on the distance between the electrodes.

The electric double layer can be described as composed of three planes or layers: the inner Helmholtz plane (IHP), named after Hermann von Helmholtz who was the first person to observe the effect of the double layer, the outer Helmholtz plane (OHP) and the diffuse layer (Figure 4.3c). The inner Helmholtz plane is closest to the electrode surface and consists of a layer of polar solvent molecules aligned with the electric field as well as specifically adsorbed ions. The outer Helmholtz plane consists of densely packed solvated ions. From the outer Helmholtz plane, a concentration gradient of additional solvated ions extends into the bulk of the electrolyte and makes up the diffuse layer.

The capacitance of an electric double layer capacitor is generally several orders of magnitude higher than that of a thin film capacitor. This is because the distance between the metal and the solvated ions is only a few Angstroms while the dielectric layer in film and ceramic capacitors is typically in the nanometer or micrometer range. 
a)

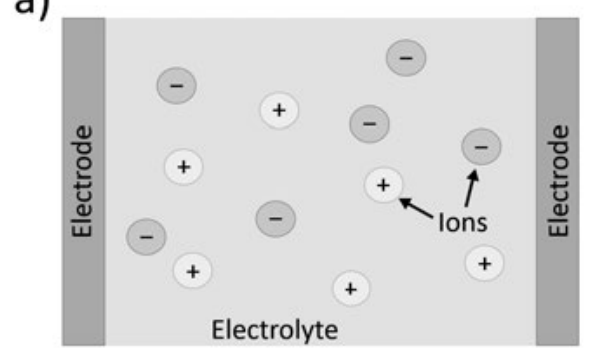

c)

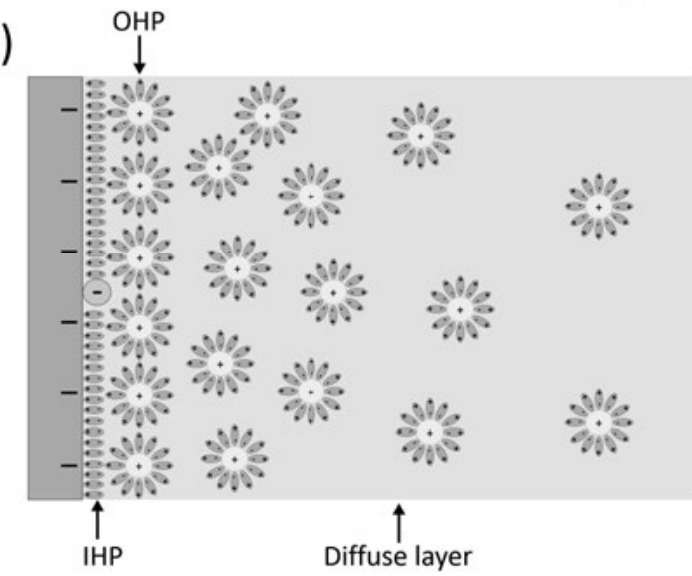

b)

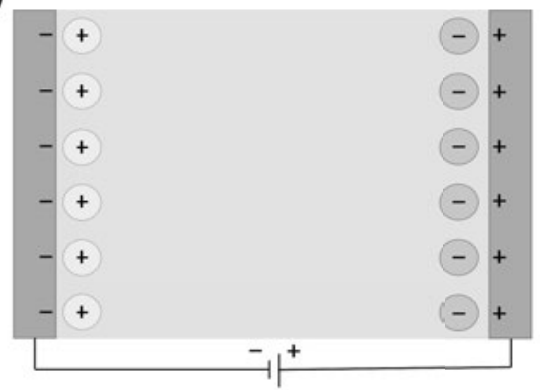

Solvated cation

Solvent dipole

Figure 4.3 Schematic of an electric double layer capacitor without (a) and with (b) an applied external voltage bias and (c) a detailed picture of the double layer.

\subsubsection{Supercapacitors}

Supercapacitors, also known as ultracapacitors, get their name from their superior capacitance compared to film and ceramic capacitors. Supercapacitors can be divided into two classes: electric double layer capacitors (EDLCs) and pseudocapacitors. Although both types are similar in design and performance, they are based on two different electronic processes. The EDLC is based on the electric double layer discussed in Chapter 4.1.2. A voltage bias is applied between two electrodes connected by an electrolyte and ions accumulate at the electrodes. A pseudocapacitor is not a capacitor by the strictest definition since it does not store charges in an electric field. Instead, the electrodes contain a material which undergoes an electrochemical reaction when a voltage is applied over the cell. Since the energy is stored as chemical energy, the principle is more like that of a 
battery. Although the microscopic processes are different, EDLCs and pseudocapacitors have similar device characteristics (see Chapter 5.4.2).

Supercapacitors, both EDLCs and pseudocapacitors, differ from other capacitors in that they usually can store charge in the bulk of the electrode, provided that there is a way for the electrolyte ions to be transported there. This can be done by using porous electrodes to increase the specific surface area or by using materials which can conduct ions. This type of volumetric capacitance makes it possible to store orders of magnitude more charge compared to flat electrodes. In the literature, capacitance is usually normalized to the volume or mass of the electrode material to evaluate its performance. These metrics are called volumetric and gravimetric specific capacitance with the units $\mathrm{F} / \mathrm{cm}^{3}$ and $\mathrm{F} / \mathrm{g}$, respectively.

Other important parameters which govern the performance of a supercapacitor are the ion intercalation, the equivalent series resistance and the physical properties of the electrolyte (such as ion mobility and concertation). Conductive polymers are ionically conductive which means ions can easily move in and out of the electrode.[96-98] Carbon and metal oxides lack ionic conductivity and instead rely on porous structures and how well the electrolyte can penetrate these pores. Porous structures can be fabricated by pressing particles together or by nanostructuring, such as nanopillars. [99] Figure 4.4 shows a schematic of electrodes with different structures. The equivalent series resistance (ESR) is the serial combination of all electrical resistances in the system. This includes the contacts, the electrodes and the electrolyte as well as interfacial resistances. A low ESR is desired since this will make the capacitor charge and discharge faster (see Chapter 4.1.1). The electrolyte has several effects on the performance of the capacitor (see Chapter 2.4). The capacitance is affected by the ionic conductivity and how well the electrolyte contacts with the porous electrode.[100] The ionic conductivity also effects the ESR. The electrochemical stability of the electrolyte determines at which voltages the capacitor can be operated. 
Porous electrode Nonostructured electrode Ionically conductive electrode
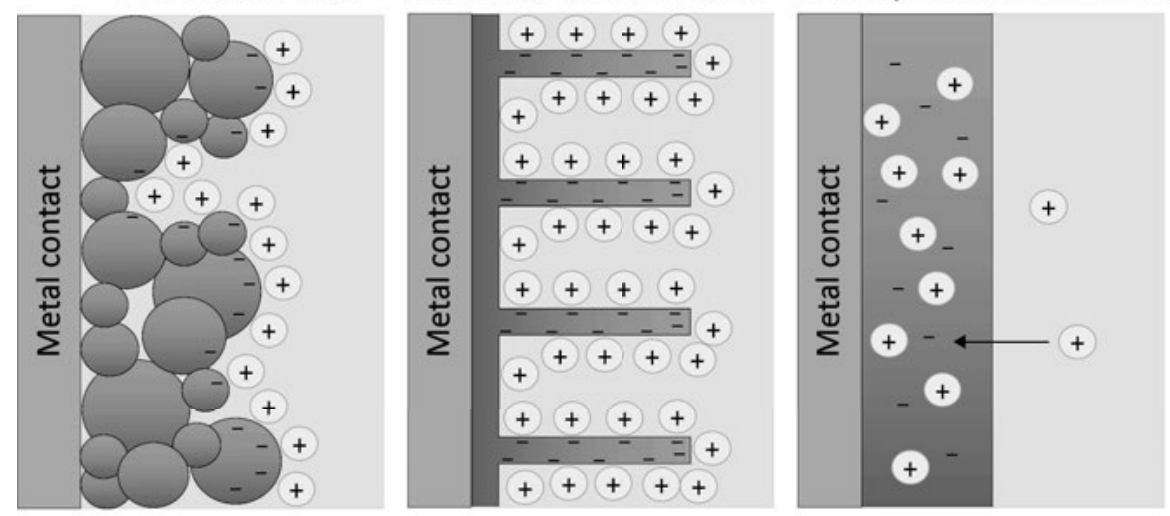

Figure 4.4 Illustration of different types of electrode structures used in supercapacitors.

Commercial EDLCs typically use carbon as the electrode material due to its low cost, chemical stability and high conductivity.[101-103] By using activated (porous) carbon, the capacitance can be greatly increased. However, porous materials also tend to have lower conductivity. Carbon materials with specific capacitance as high as $400 \mathrm{~F} / \mathrm{g}$ has been reported in the literature. ${ }^{[32]}$ However, in commercial supercapacitors the values are typically one order of magnitude lower.[104]

Pseudocapacitors are often based on either conductive polymers or transition metal oxides.[101-102] Conductive polymers can be processed in solution form at room temperature, they have good conductivity and high mechanical flexibility. Typical reported values of capacitance based on conductive polymers is in the range 10-200 F/g. [7, 39, 102] Some metal oxides, like ruthenium oxide, have superior capacitance compared to both carbon and conductive polymers with a specific capacitance $\sim 1000 \mathrm{~F} / \mathrm{g} .{ }^{[99]}$ However, they often suffer from low conductivity and poor ion permeability. Both conductive polymers and metal oxides also suffer from short lifetime compared to EDLCs and are more expensive materials than carbon. As a result, there are few commercial pseudocapacitors on the market.

\subsubsection{Hybrid Supercapacitors}

There are many ways of storing energy to be used in different applications. Most vehicles still use the chemical energy stored in fossil fuels and convert this energy to mechanical energy in a combustion engine. Electric cars use either batteries or fuel cells, which are greener alternatives to fossil fuels. In electronics, capacitors, supercapacitors or batteries are typically used for energy storage. 
Which type of device is used for a specific application depends on the differences in device parameters. Two of the most important device parameters for energy storage devices are the specific energy (or energy density), typically measured in $\mathrm{Wh} / \mathrm{kg}$ and specific power (or power density) measured in $\mathrm{W} / \mathrm{kg}$. Specific energy gives a measure of the amount of energy which can be stored in a device of a certain mass and the specific power is a measure of how fast the device can store and release this energy. Figure 4.5 shows the specific energy and power of different technologies in a Ragone plot. The dashed lines correspond to different charging/discharging times of the technologies intersecting the lines. There is a factor 10 difference between each line.

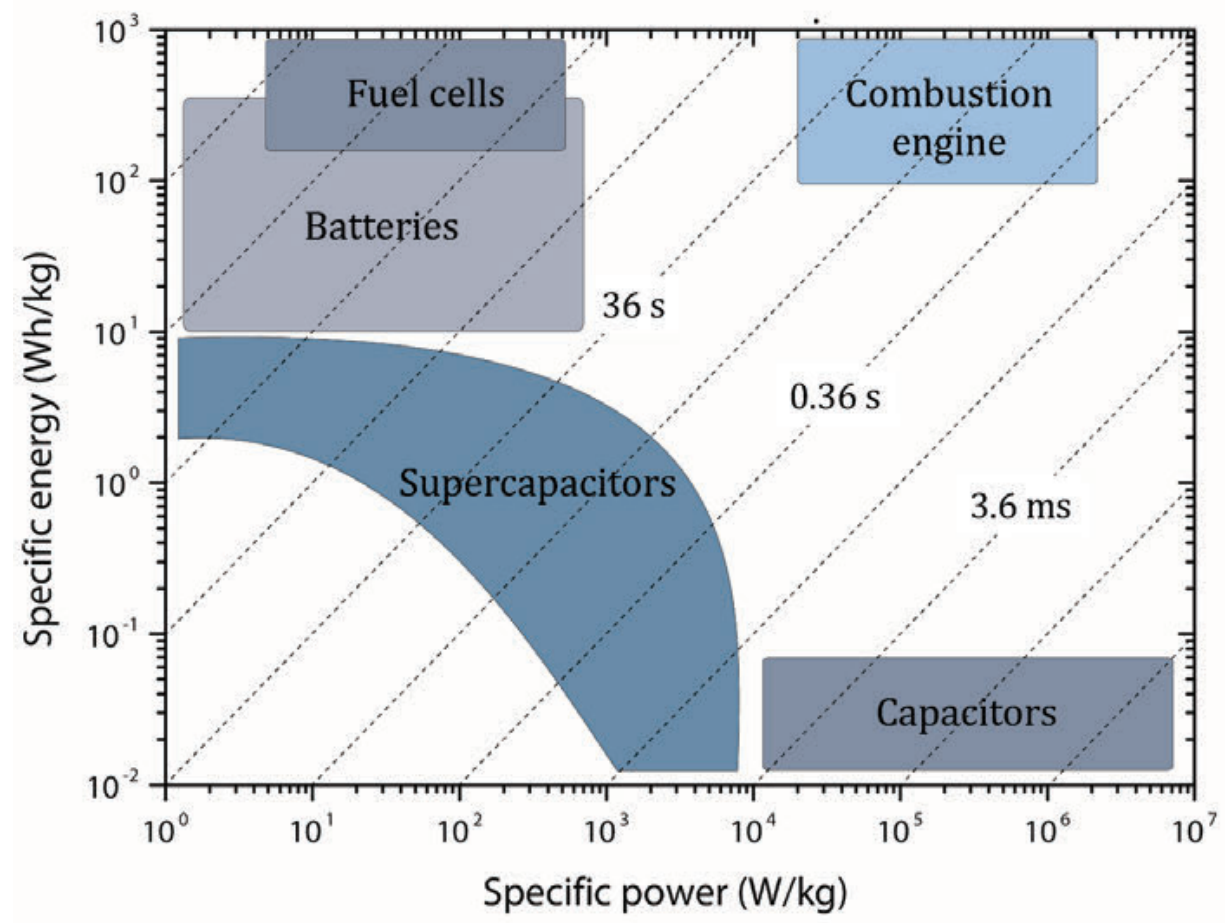

Figure 4.5 Ragone plot of different energy storage technologies.[105-106]

As is evident from Figure 4.5, capacitors, supercapacitors and batteries each dominate one area of the Ragone plot. Among these three categories, batteries are superior in terms of energy storage. For rechargeable batteries, lithium ion batteries (LIB) are one of the best performing types in terms of combined energy and power density and are commonly used in portable electronic devices such as computers and smartphones. However, the charging time of a battery is typically 40 
more than 1 hour, which limits their use for applications such as electric vehicles where fast charging is desired. Supercapacitors are interesting candidates for energy storage applications due to their higher specific power compared to batteries. Also, supercapacitors generally have a better cycling life than most rechargeable batteries. However, supercapacitors have too small specific energy to be used in the type of technologies that are dominated by batteries. To improve the energy density of supercapacitors while maintaining high power density, different types of hybrid supercapacitors have been developed. By combining materials with fast charge transfer kinetics, such as carbon or PEDOT, with materials with high specific energy, such as transition metal oxides, redox active organic molecules or lithium compounds, the overall device performance can be improved.

Vlad et al. reported a hybrid supercapacitor-battery system by compositing lithium iron phosphate $\left(\mathrm{LiFePO}_{4}\right)$ with the conductive polymer poly(2,2,6,6tetramethyl-1-piperinidiloxy-4-yl methacrylate) (PTMA).[107] The fast charge transfer kinetics and ion mobility of the conductive polymer combined with the large specific capacitance of $\mathrm{LiFePO}_{4}$ resulted in superior performance compared to the individual components. Similarly, Zhang et al. combined iron oxide $\left(\mathrm{Fe}_{3} \mathrm{O}_{4}\right)$ with graphene in a LIB to enhance charge transfer kinetics.[108] Also, the combination of conductive polymers, such as PEDOT and PPy, with small organic molecules with redox active phenolic groups have resulted in enhanced charge storage capacity compared to the polymers alone.[39-40,42, 109]

\subsection{Organic Electrochemical Transistors}

Transistors are three terminal devices used in electronics for switching and/or amplifying signals. The current between two of the terminals, commonly known as source $(S)$ and drain $(D)$, is controlled by changing the electrical potential on (or current through) the third terminal, known as the gate $(G)$. Other terminologies are sometimes used for the terminals depending on the type of transistor. Common to all transistors is that they involve the modulation of the conductance of a semiconducting material. One common type of transistor is the field effect transistor (FET). The source and drain terminals are connected by a semiconducting path known as the channel, and the channel is separated from the gate by a dielectric. For a transistor working in enhancement-mode, the channel originally has a low conductance. Upon applying a voltage bias at the gate, charges will be injected into the channel from the contact to compensate for the charge on 
the gate, similar to the charging of a capacitor. The injected charges form a conductive layer which enhances the conductance of the channel and the transistor is said to "turn on". The transistor can also be designed to operate in depletionmode and "turn off" when applying a voltage to the gate. Although inorganic semiconductors are typically used in FETs, organic semiconductors are also possible. These transistors are known as organic field effect transistors (OFET). The dielectric in an OFET can further be replaced by an electrolyte, resulting in an enhanced capacitance and lower operating voltage. This type of transistor is known as an electrolyte gated field effect transistor (EGOFET).

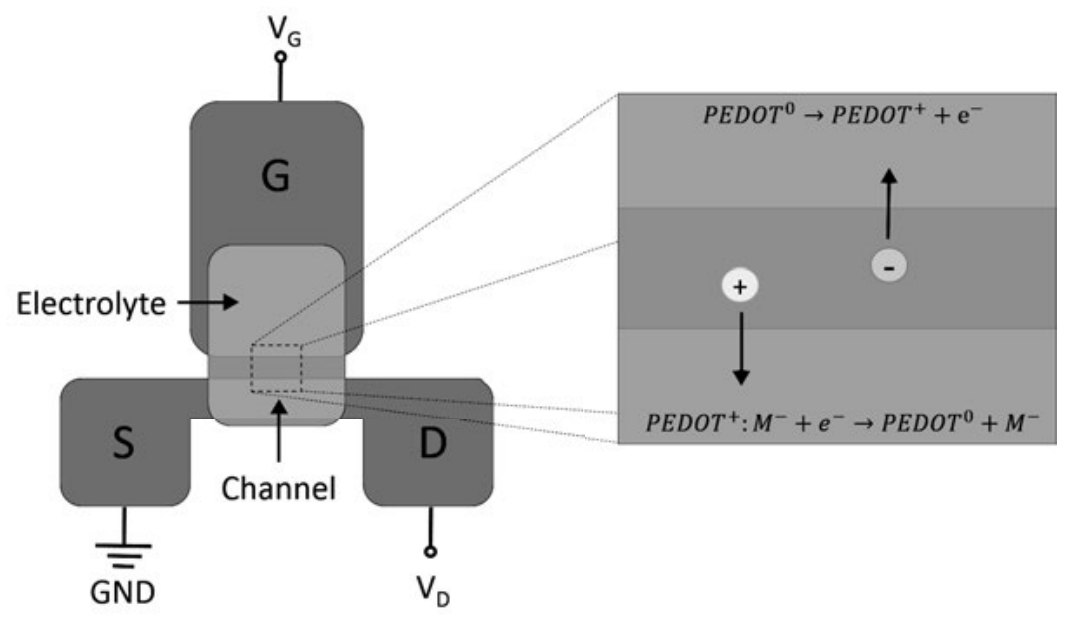

Figure 4.6 Schematic of an organic electrochemical transistor with lateral design.

The organic electrochemical transistor (OECT) is usually based on the conductive polymer PEDOT and have many design similarities with the FET, OFET and EGOFET. Like the EGOFET, an electrolyte is used instead of a dielectric. However, the modulation of the channel conductance is not caused by the field effect, which is a surface effect. Instead, ions from the electrolyte can move in and out of the bulk of the channel and induce an electrochemical reaction. Upon applying a positive voltage to the gate, the PEDOT in the channel undergoes a reduction reaction which lowers the conductivity. This means that the transistor is operating in depletion-mode. Figure 4.6 shows the schematics of a lateral OECT with source, drain and gate in the same plane.

OECTs have been used in logical circuits in printed electronics. ${ }^{[56]}$ However, due to their slow switching speed, they have limited application in digital electronics. One area where OECTs have demonstrated superior properties is for 
sensors, such as biosensors. One of the most important parameters in transistors used for sensor applications is their transconductance $\left(g_{m}\right)$. The transconductance, defined in Equation 4.4, is the rate of change of the drain current $\left(I_{D}\right)$ with respect to the gate voltage $\left(V_{G}\right)$ and is a measure of a transistors ability to amplify a signal. Therefore, a high transconductance will result in a high sensitivity when a transistor is used as a sensor. The transconductance is further related to the capacitance ( $C_{c h}$.) of the channel. The use of an electrolyte in EGOFETs is to increase the capacitance and therefore the transconductance. However, due to the volumetric capacitance of PEDOT[110], the capacitance can reach even higher values of transconductance compared to EGOFETs. ${ }^{[111]}$ Rivnay et al. demonstrated how the transconductance in an OECT can be tuned by the thickness of the device and used these transistors to measure human brain rhythms. ${ }^{[12]}$

$$
g_{m}=\frac{\Delta I_{D}}{\Delta V_{G}} \propto C_{c h}
$$

\subsection{Thermoelectric Generators}

Thermoelectric generators (TEG) are devices which can harness electrical energy from thermal energy once subjected to a temperature difference. These devices have been used to drive sensor systems and radio communication in remote areas which are difficult to reach for service. One such example is space probes where the thermal gradient comes from a radioactive isotope.[113] Such a system does not have any moving parts, is neither affected by day-night cycle or dust/sand coating, so it is very robust and can survive for long times. Thermoelectric generators have also been envisioned to harvest waste heat in applications such as factory machines, cars and power plants.[114]

The most efficient thermoelectric materials to date are inorganic highly doped semiconductors such as bismuth telluride ( $\left.\mathrm{Bi}_{2} \mathrm{Te}_{3}\right)$ and its alloys. ${ }^{[115]}$ However, most of the promising thermoelectric materials are either rare, and therefore expensive, or operate at high temperatures.[116-117] For these reasons, there is a growing interest in TEGs based on organic materials. Organic semiconductors such as PEDOT:PSS are inexpensive and have a thermoelectric effect which can be harnessed at room temperature with small temperature gradients. Furthermore, due to the ease of processing and inherent flexibility of conductive polymers, organic-based TEGs can be used in applications such as printed electronics, etextiles and wearable electronics.[118-119] 


\subsubsection{The Seebeck Effect}

The thermoelectric effect, the direct conversion of thermal to electrical energy, can be subdivided into three different effects: the Peltier effect, the Thomson effect and the Seebeck effect. The Seebeck effect, named after Thomas Johann Seebeck who was the first person to observe the phenomenon, is caused by a difference in the population of charges at different energies in a conductor with a temperature gradient.[120] The population of charge carriers in a conductor is governed by the Fermi-Dirac distribution. The Fermi function $(F)$, given in Equation 4.5, shows how the relative population changes with temperature. Here, $\epsilon$ is the energy, $\mu$ the chemical potential, $k_{B}$ the Boltzmann constant and $T$ the temperature. With increasing temperature, there will be a higher population of charges at high energy, resulting in a higher average kinetic energy.
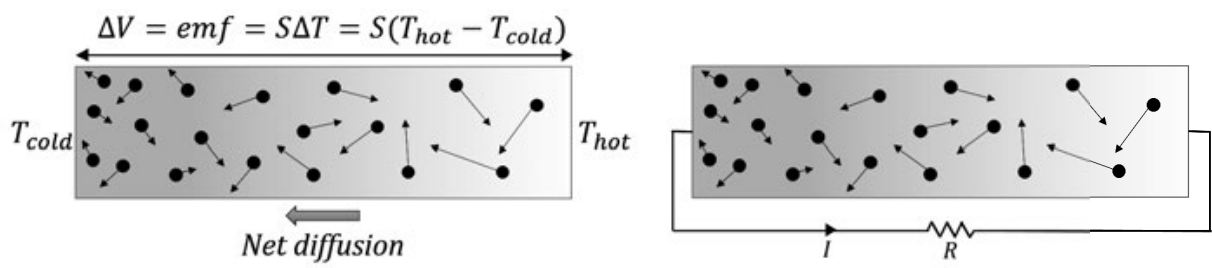

Figure 4.7 Schematic illustration of the Seebeck effect in a conductor with a temperature gradient.

Figure 4.7 illustrates a conductor with a temperature gradient. At the warmer end, carriers have a higher average velocity due to the higher energy. Diffusion of charge carriers will result in a net charge transport from the hot side to the cold side. As the charge carrier concentration builds up on the cold side, an electromotive force (emf) is induced which counteracts further transport. At steady state, when the net current is zero, the emf is given by Equation 4.6, where $\Delta T$ is the temperature difference and $S$ is the Seebeck coefficient, which is a material parameter. If the conductor is connected to an external load, the emf can be used to drive a current, as illustrated by Figure 4.7, where the amount of power delivered to the load will depend upon $\mathrm{S}$ and $\Delta \mathrm{T}$ as shown in Equation 4.7.

$$
\begin{aligned}
& F(\epsilon)=\frac{1}{e^{\frac{\epsilon-\mu}{k_{B} T}}+1} \\
& e m f=V=-S \Delta T
\end{aligned}
$$




$$
P=I^{2} R=\frac{V^{2}}{R}=\frac{S^{2} \Delta T^{2}}{R}
$$

\subsubsection{Energy Harvesting with TEGs}

The ability of a material to produce electrical power by the Seebeck effect is often quantified using the dimensionless figure of merit, ZT. Equation 4.8 defines the figure of merit where $\sigma$ is the electrical conductivity, $\lambda$ is the thermal conductivity, $S$ is the Seebeck coefficient and $T$ is the temperature. From this equation, it becomes clear that to optimize the power output, one wants a material with high Seebeck coefficient, high electrical conductivity and low thermal conductivity.

$$
Z T=\frac{\sigma S^{2} T}{\lambda}
$$

A low electrical conductivity will result in poor charge transport and a high thermal conductivity will dissipate the thermal gradient. Metals have high electrical conductivity, but also high thermal conductivity, and are therefore not suitable as thermoelectric materials. Polymers generally have low thermal conductivity due to their amorphous structure. Therefore, conductive polymers are good candidates as thermoelectric materials.

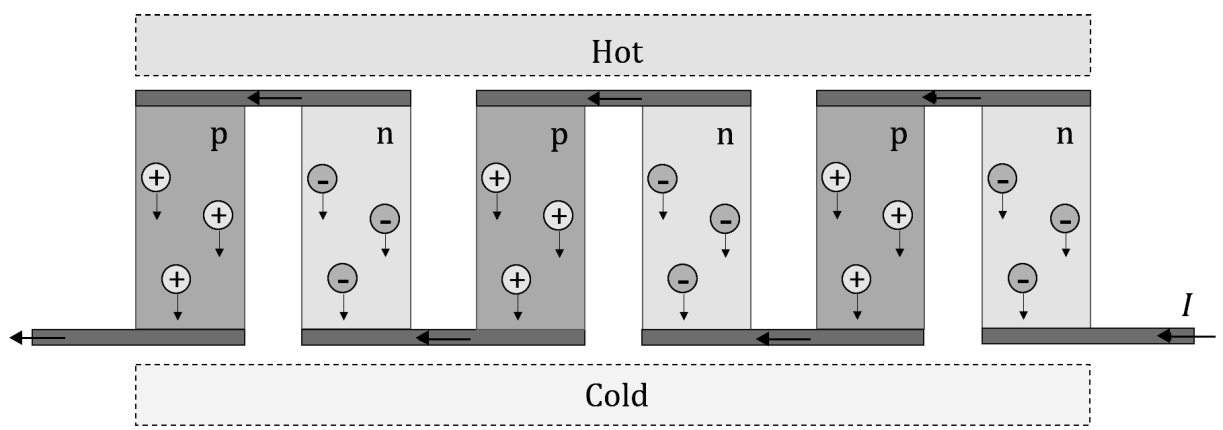

Figure 4.8 A thermoelectric module with three thermocouples connected in series.

For efficient thermoelectric energy harvesting, a so called thermoelectric module is needed. This type of structure, illustrated in Figure 4.8, consists of several thermocouples in series, connected by metal interconnects. The thermocouple consists of two "legs", one with a p-type semiconductor and one with an n-type semiconductor. In the p-type semiconductor leg, the electrical potential will be higher on the cold side of the device where positive charges accumulate. 
However, in the n-type material the higher electrical potential will be on the hot side where negative charges are being depleted. This means that the voltage over the p- and n-type legs will be added together. With this type of structure, it is possible to build up the voltage over each added thermocouple and thereby multiply the power output.

\subsubsection{Dual Sensors}

If the Seebeck coefficient of a material is known, then measuring a voltage across a piece of the material can be used to determine a temperature difference. In this way, a thermoelectric material can be used as a temperature sensor. If the temperature on one side is known and constant, then a measure of absolute temperature can be determined for the opposite end of the device. The resistance of the material is directly proportional to its length, and if the material is flexible, then a mechanical deformation can result in a change in resistance.
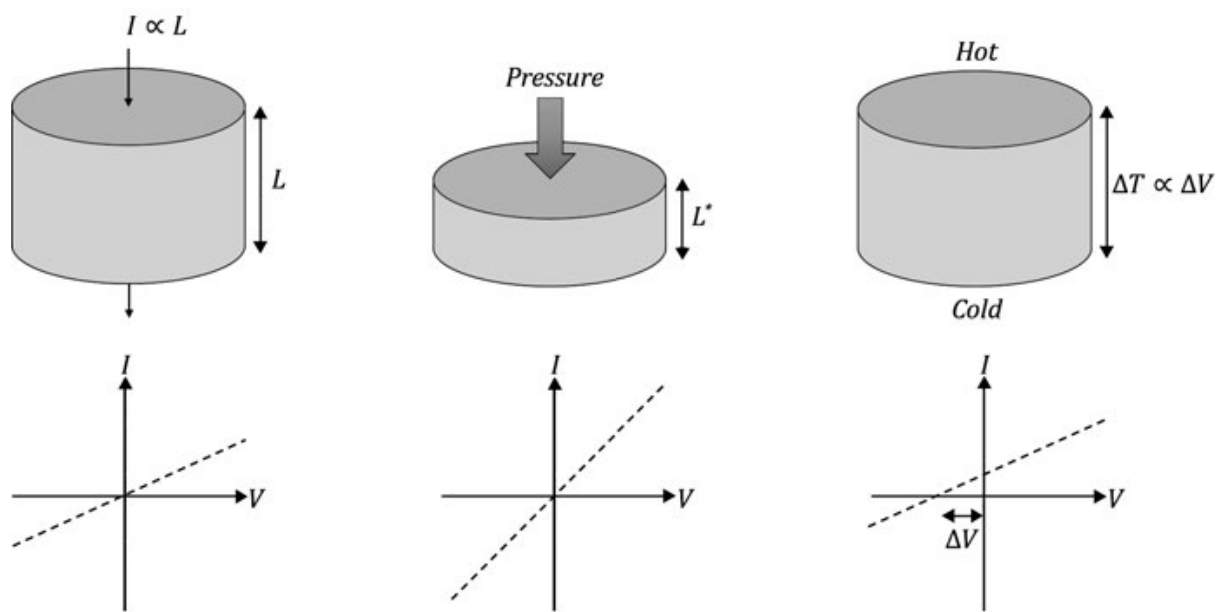

Figure 4.9 The concept design of a dual pressure and temperature sensor.

Figure 4.9 shows how a flexible thermoelectric material can be used as a dual sensor, measuring both pressure and a temperature difference. When a voltage is applied across the conductive material, a current will run through it. The current will be inversely proportional to the length of the conductor. When a pressure is applied, the material is compressed and the length decreases resulting in an increase in current, as can be seen by the slope in the IV-curve. If a temperature gradient is applied across the material, a voltage will build up due to the Seebeck effect. This will result in a shift in the IV-curve. Both pressure and temperature can 
therefore be measured simultaneously by observing the slope and shift of the IVcurve.

\subsection{Electrochromic Devices}

Electrochromism is the ability of a material to change color during a redox reaction. An electrochromic device (ECD) typically consists of an electrochemical cell with the active material coated on one or both electrodes. The cell, depicted in Figure 4.10, consists of two electrodes separated by an ion conductor. Usually, at least one of the electrodes is a transparent conductor, like indium tin oxide (ITO), on transparent glass or plastic. The counter electrode consists of a conductor (transparent in case of smart windows) with a layer which can balance the reaction in the electrochromic film. This can be a second electrochromic material or a porous conductor for double layer charging. When a voltage is applied across the cell, the active material is reduced or oxidized (depending on the polarity of the voltage). Ions either move from the electrolyte into both films, or from the counter electrode to the active layer. In the second case, the electroactive layer on the counter electrode is referred to as an ion storage layer.

Some applications of ECDs include smart glass and electrochromic displays. ${ }^{[121]}$ Smart glass refers to fully transparent devices which can modulate the light throughput at certain wavelengths. These devices are being used in the windows of buildings or vehicles to either dim the windows or to regulate heating by changing the throughput in the infrared region of the electromagnetic spectrum.

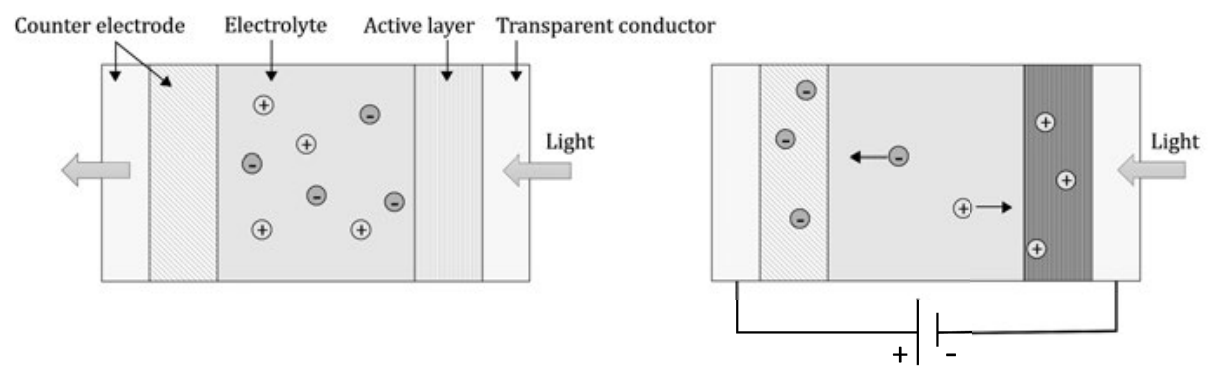

Figure 4.10 General structure of an ECD in its transmitting (left) and blocking (right) state.

There are three main categories of electrochromic materials: transition metal oxides, organic materials such as conjugated polymers and intercalated materials. Many conductive polymers, like PEDOT:PSS, exhibit electrochromism in the visible 
spectrum. Due to the ease of processability and flexibility of conductive polymers, they have been widely used as the active material in printed and flexible ECDs. ${ }^{[46}$, 122-123] PEDOT switches from a transparent state to a deep blue color upon electrochemical reduction. In this state, the main absorption peak is in the red region of the visible spectrum while blue light is transmitted. In ECDs that are used as optical shutters, it is desirable to go from a state of high transmittance to low transmittance over the entire visible spectrum. However, most electrochromic materials only absorb in part of the visible spectrum. By using complementary materials, the absorption band in the dark state can be widened. Two materials are said to be complementary if one becomes dark (absorb in the visible spectrum) upon reduction and the other dark upon oxidation. In this way, the two complementary materials can be coated on opposite electrodes in the electrochemical cell and will go dark/transparent at the same time when applying a voltage. One example of complementary materials is PEDOT and PPy. PEDOT absorbs strongly in its reduced state while PPy absorbs in its oxidized state. 


\section{Methods}

\subsection{Solvent Casting and Spin Coating}

There are many ways of preparing polymer films from solution form such as printing, dip coating, drop casting, solvent casting and spin coating. The solution either contains a dissolved polymer or a suspension of polymer particles/droplets. Spin coating is a simple method of preparing thin films on small scale substrates. A substrate is attached to a spinner by a vacuum suction and a solution containing the polymer is added to the substrate. The substrate is then spun at high rotation (typically 1000-3000 rpm) to remove excess solution. A thin liquid layer with uniform thickness will remain on the substrate. The thickness of the layer will depend on the spinning speed, the viscosity of the solvent and the surface energy between the substrate and the solution. The final thickness of the sample depends on the thickness of the liquid layer after spinning and the concentration of the polymer in the solution.

An even more straight forward way of producing polymer films is by solvent casting. In its simplest form, a solution containing the polymer is cast into a container, such as a petri dish, after which the solvent will evaporate, leaving only the polymer film. The thickness of the sample is easily controlled by the volume of the solution if the concentration and density of the polymer is known.

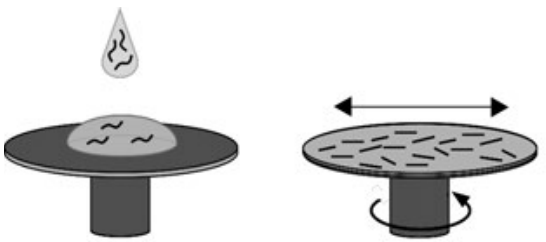

Spin coating
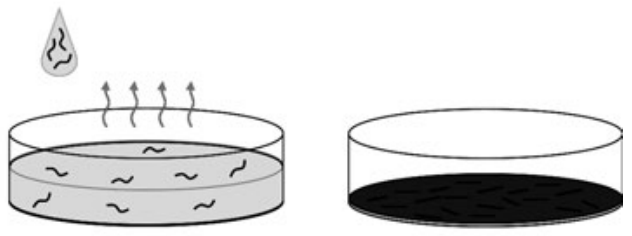

Solvent casting

Figure 5.1 The principles of spin coating (left) and solvent casting (right) polymer films from solution.

\subsection{Vapor Phase Polymerization}

The process of producing polymers from their molecular building blocks, monomers, is known as polymerization. There are a number of different polymerization methods, including electropolymerization (also called 
electrochemical polymerization ${ }^{[124]}$, chemical polymerization ${ }^{[125]}$ and vapor phase polymerization (VPP) ${ }^{[126]}$. All methods involve the oxidative radicalization of the monomers which causes them to form covalent bonds with each other. In electropolymerization this is achieved by applying a positive electrical bias to a conductive substrate in a solution containing the monomer. This method offers a great deal of control over the polymerization reaction since the current or potential bias can be controlled by a source meter or a potentiostat. The amount of produced polymer can also be controlled by integrating the current to obtain the charge which will be proportional to the number of reacted monomers. However, electropolymerization can only produce polymer films on conductive substrates which limits its range of applications.

Chemical polymerization and VPP are based on the same principle. Instead of an electric bias, a strongly oxidative chemical (oxidant) is used to produce the monomer radicals. A commonly used oxidant is $\mathrm{Fe}^{3+}$ coupled with co-ions such as chloride or tosylate. ${ }^{[127-130]}$ In chemical polymerization, the oxidant and monomer are mixed in a solution. The solution can then be coated on a substrate, for example by spin coating, followed by heating of the substrate, resulting in a polymer film. In VPP, a solution containing the oxidant (the oxidant solution) is first coated on a substrate. The substrate is then put in a vacuum chamber where it is exposed to vapor of the monomer which is evaporated from a heater. The monomer will condense on the substrate and react with the oxidant. This method offers a greater control over the polymerization rate compared to chemical polymerization. Thus, very high values of conductivity have been reached using VPP. Conductivity as high as $5400 \mathrm{~S} / \mathrm{cm}$ has been realized for thin films ${ }^{[9]}$ and $>7000 \mathrm{~S} / \mathrm{cm}$ in nanowires ${ }^{[131]}$ have been reported for the conductive polymer PEDOT.

Figure 5.2 shows the chemical reactions occurring during VPP of PEDOT as well as the setup of the VPP chamber. The monomer (1), in this case EDOT, becomes oxidized by $\mathrm{Fe}^{3+}(2)$. This results in the formation of a dimer (3-4) followed by deprotonation (5). The process is then repeated (6-7) with increasing length of the polymer. There are different theories regarding how the deprotonation happens, but evidence suggests that water is a main contributor, resulting in the formation of hydronium ions. ${ }^{[132-133]}$ Further oxidation by the $\mathrm{Fe}^{3+}$ results in the doping of PEDOT. Anions in the oxidant solution (the co-ions to $\mathrm{Fe}^{3+}$ ) coordinate with PEDOT to compensate the positive charges on the backbone. The polymerization is first initiated at the surface of the oxidant film. New oxidant is then transported through the film to the surface as the film grows in thickness. ${ }^{[134]}$ This means that VPP is a bottom-up process initiated at the surface. After the 50 
polymerization is done, the sample is washed in a solvent like water or ethanol to remove unreacted oxidant solution.

(3)
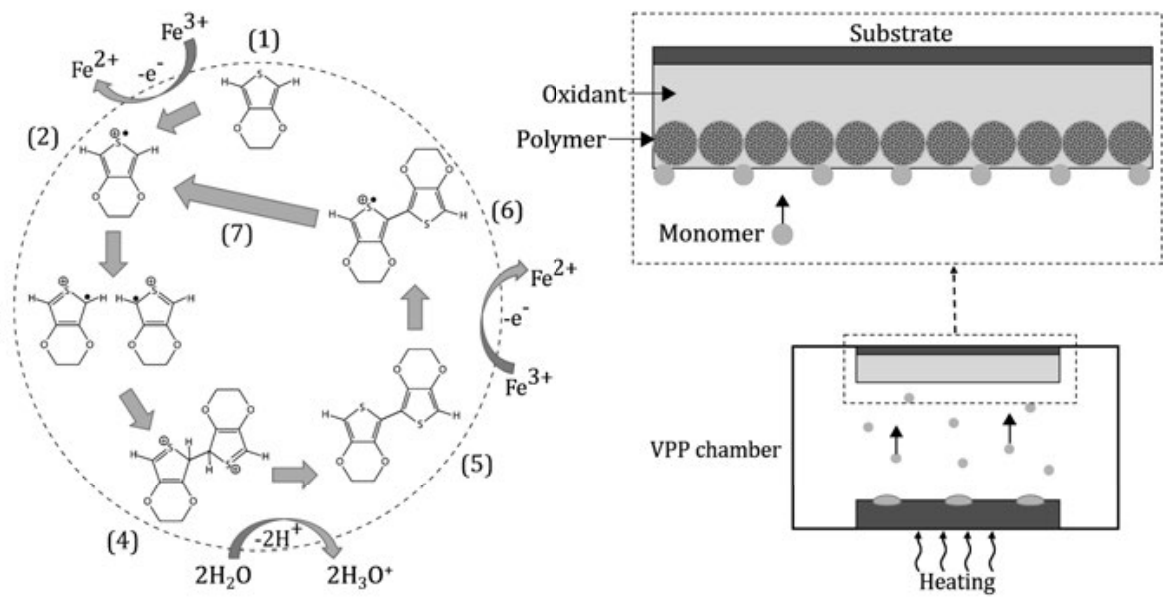

Figure 5.2 The polymerization process (left) and experimental setup (right) of VPP of PEDOT using an iron oxidant.

\subsection{Freeze-drying}

Freeze-drying is a method of removing a solvent from a solution containing a solid material, such as a polymer, by sublimation. When a polymer suspension in liquid water dries, strong intermolecular forces between the polymer and water will act to collapse the polymer into a dense structure as water evaporates. Freezedrying offers a way to avoid this collapse and preserve the structure that the polymer had in solution form. The sample is first frozen and then put in a vacuum chamber. As the pressure decreases, it will cross the solid-gas line in the water phase diagram where water is sublimated and never transitions into liquid form. The transitions in the phase diagram is shown by the arrows in Figure 5.3. The figure also shows the freeze-drying procedure.

Freeze-drying is used in the pharmaceutical industry and the food industry to dehydrate products for improved shelf life. To reach the same level of hydration without freeze-drying, high temperatures are often necessary which can damage the product. Freeze-drying is also used in science to manufacture highly porous and lightweight materials, so called aerogels. A hydrogel is a polymer gel swollen by water. It can contain large amounts of water while remaining solid or semi-solid. In the hydrogel, the polymer forms a continuous porous network. When the water 
evaporates, the pores collapse from the surface tension. By freeze-drying the hydrogels, the water can be removed without damaging the network, resulting in low density aerogels.

Recently, aerogels based on cellulose and chitosan have received a lot of attention due to their mechanical strength and flexibility. These structures have in some cases been further functionalized with other materials for use in various applications. ${ }^{[29,135-136]}$ However, non-polymeric materials such as carbon and silica are also often used.[31] In the literature, aerogels have been used in a wide range of applications such as: biomedical application, such as for drug delivery and tissue regeneration [31], water cleaning and oil absorption ${ }^{[137-138]}$, thermal insulation ${ }^{[30,139-}$ 140] and supercapacitors ${ }^{[32,141-143]}$.
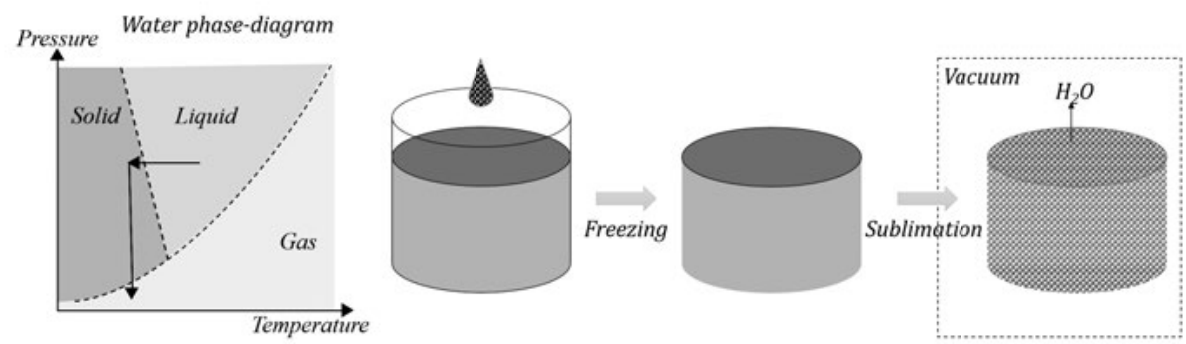

Figure 5.3 Phase diagram of water (left) and the freeze-drying procedure (right).

\subsection{Electrochemical Characterization}

Electrochemistry is the study of the connection between chemical reactions and electrical current. This encompasses both chemical reactions caused by the passage of a current (electrolytic reactions) and electrical current caused by a chemical reaction (galvanic reactions). Electrochemistry has many industrial applications such as chemical production and electroplating and has resulted in many types of electrochemical devices such as batteries, fuel cells and sensors. In science, electrochemistry is a valuable tool for analysis of various chemical reactions and systems as well as device characterization.

\subsubsection{The Electrochemical Cell}

An electrochemical system comprises at least two electrodes separated by an electrolyte (ionic conductor) and is called an electrochemical cell. When an external voltage bias is applied between the two electrodes, electrons can pass 
between the electrodes and molecules in/on the electrodes. The removal of an electron from a molecule is known as oxidation while adding electrons is called reduction. A minimum of two electrodes is necessary since any reaction occurring at one electrode must be balanced by a reverse reaction on the opposite electrode to maintain charge neutrality in the cell. That is to say, if oxidation is occurring at one electrode, transporting electrons from the system, a reduction reaction must occur on the opposite electrode to supply new electrons to the cell. Reductionoxidation reactions are also known as redox reactions, and the two species involved in a reaction are known as a redox couple. Figure 5.4 shows energy diagrams of a molecule close to an electrode. Initially, the electron energy, or Fermi level $\left(E_{F}\right)$, of the metal lies in between the HOMO and LUMO levels of the molecule. At this point, there is no charge transfer. If the potential of the electrode is sufficiently decreased, the energy of the electrons will increase and the Fermi level goes above the LUMO level. At this point, electrons can be transferred to the empty LUMO level to obtain a lower energy state. This is a reduction reaction. Likewise, if the potential of the electrode is raised then the electron energy will eventually fall below the HOMO level and electrons can be transferred from the molecule to the electrode. This is an oxidation reaction.
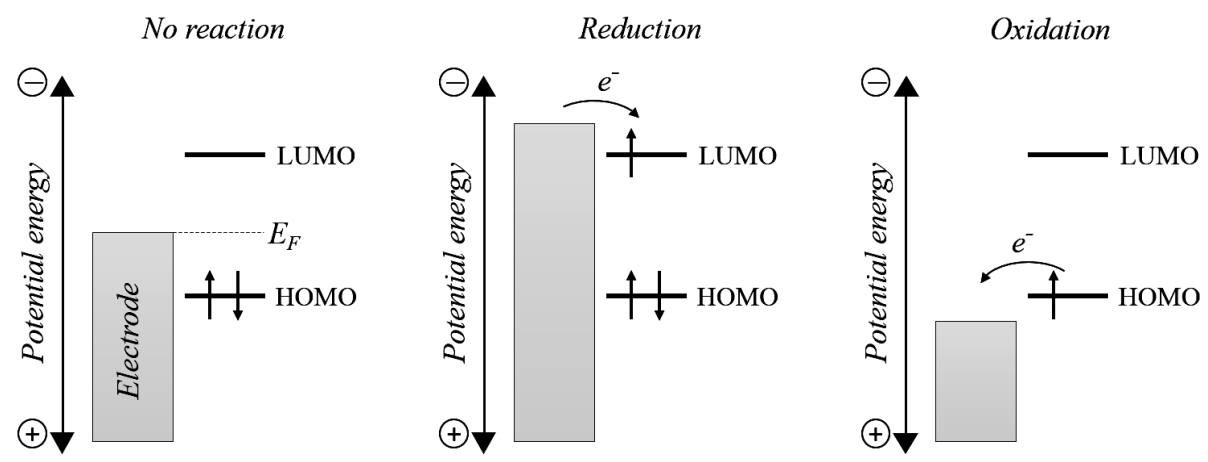

Figure 5.4 Energy diagrams showing charge transfer reactions between an electrode and a molecule.

Redox reactions, also known as Faradic reactions, involve the transfer of electrons between the electrodes and the electrolyte. However, each reaction only happens at certain potentials, known as their standard potential $\left(E^{0}\right)$. This means that, depending on the electrolyte and electrode material, for certain potential ranges there will be no Faradic reaction. Even though no reactions are occurring, it is still possible for a current to run through the external circuit. This is because ions in the electrolyte become attracted to the charged electrodes, resulting in the 
formation of a double layer (Chapter 4.1.2). The electrode is in this case said to be polarized. An ideally polarizable electrode will never pass a Faradic current no matter what the electrical potential. Instead, an increasingly large voltage drop will build up at the electrode-electrolyte interface. Likewise, an ideally non-polarizable electrode is an electrode which can pass an electrical current between the electrode and the electrolyte without any resistance and will therefore never have a potential drop at the electrode-electrolyte interface. In reality, neither an ideally polarizable nor an ideally non-polarizable electrode exist, but certain electrodeelectrolyte systems approach the ideal cases over certain potential ranges.

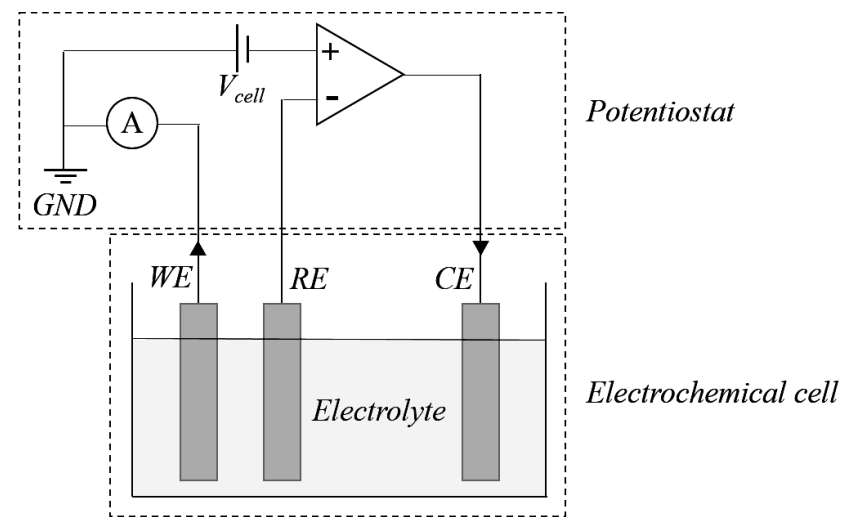

Figure 5.5 Schematic structure of a three-electrode electrochemical cell and a potentiostat.[144]

An electrode which works close to an ideally non-polarizable electrode is of special interest for electrochemical measurements. If one such electrode is used in an electrochemical cell, then any change in the potential between the two electrodes must have occurred at the other electrode. This is the principle of a reference electrode. The equipment commonly used to study electrochemical systems in called a potentiostat. A potentiostat is an electronic circuit with a power supply which can control the electrical potential at an electrode. Reactions in a system can be studied by applying a perturbation and monitor the response. For example, the potential can be changed while monitoring the current through the cell. Reference electrodes $(R E)$ are used as points of fixed and known potential. The potential of an electrode under investigation, often called the working electrode $(W E)$, can be determined by measuring the potential difference between the RE and $W E$. There are several commonly used reference electrodes such as the normal hydrogen electrode $(N H E)$, standard calomel electrode $(S C E)$ and $\mathrm{Ag} / \mathrm{AgCl}$ electrode. The $N H E$, which involves the oxidation and reduction of the $\mathrm{H}^{+} / \mathrm{H}_{2}$ redox 54 
couple has a standard potential close to $\mathrm{E}^{0}=0 \mathrm{~V}$. This is because the potential of the hydrogen redox reaction has been defined as $0 \mathrm{~V}$. The standard potentials of all other reactions are measured with respect to the $\mathrm{H}^{+} / \mathrm{H}_{2}$ redox couple.

Figure 5.5 shows the structure of a three-electrode electrochemical cell and the simplified circuitry of a potentiostat. The third electrode is called the counter electrode $(C E)$ or auxiliary electrode and is used to avoid the effect of a large ohmic drop in the electrolyte when measuring at high current levels, which can otherwise result in an incorrect estimation of the potential at the WE. The $C E$ supply the counter-charge to the $W E$ while a negligible current pass through the $R E$ resulting in a small ohmic drop between the $R E$ and $W E$. A platinum wire or mesh is often used as $C E$ due to the low chemical reactivity of platinum. The role of the potentiostat is to make sure that the potential at the $R E$ is equal to $V_{\text {cell. }}$ This is done by supplying the necessary current to the $C E$. However, the potential at the $C E$ is unknown during the experiment. The small ohmic drop between the $W E$ and $R E$ can be minimized by placing these electrodes close to each other.

\subsubsection{Cyclic Voltammetry}

Cyclic voltammetry is a potential sweep method used to characterize electrochemical systems. The WE potential is swept at a constant scan rate $v$ (with the unit $\mathrm{V} / \mathrm{s}$ ) while monitoring the current. The formal name of this type of method is linear potential sweep chronoamperometry. When the potential is swept from a potential $E^{1}$ to a potential $E^{2}$ and then back to $E^{1}$, this is called cyclic voltammetry. Figure 5.6 shows the potential sweep vs. time as well as two cyclic voltammograms. The middle graph shows the result of a potential sweep of an electrochemical capacitor. This could be either an electric double layer capacitor or a pseudocapacitor. An ideal capacitor would have a perfectly rectangular shape, although any real system will show some deviation from ideal behavior. As the potential is swept, the current first increases rapidly and then plateaus to some constant current value $I^{*}$. The capacitance $(C)$ of the electrode can be calculated according to Equation 5.1.

$$
C=\frac{I^{*}}{v}
$$

The right graph in Figure 5.6 shows the characteristic voltammogram of a redox reaction of the type which was described in Figure 5.4. The voltammogram has two peaks at two different potentials: the anodic peak potential $\left(E_{p a}\right)$ and the cathodic peak potential $\left(E_{p c}\right)$. The formal potential $E^{0^{\prime}}$, which often replaces the 
standard potential $\left(E^{0}\right)$ and includes some corrections to the system under nonstandard conditions, lies just between the anodic and cathodic peak potentials. During the anodic (forward) sweep, the potential is initially below the formal potential. As the potential reaches the formal potential the current starts to increase as the reactant becomes oxidized. The current will continue to increase until the reactant close to the electrode has been depleted. The current then drops and plateaus to a constant value. At this point the current is limited by mass transfer of the reactant to the electrode. In the cathodic (reverse) sweep, the opposite reaction will occur (reduction) resulting in the cathodic peak. Cyclic voltammetry can hence be used both as a way of determining the nature of the reaction (capacitive or redox) and give information about redox potentials.
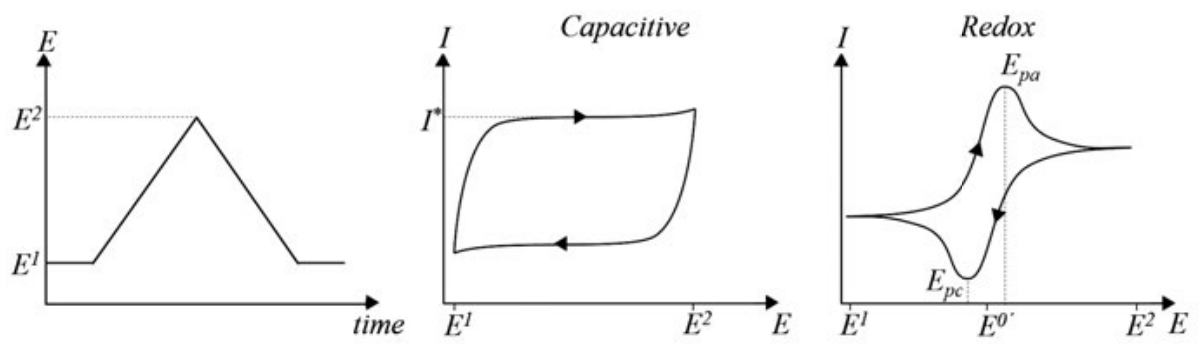

Figure 5.6 Cyclic voltammograms for capacitive and Faradic/Redox reactions.

\subsubsection{Chronopotentiometry}

Chonopotentiometry is a method where the potential of an electrode is monitored over time. This can be done in a galvanostatic measurement when a constant current bias is applied while measuring the change in potential. Figure 5.7 shows different response to the potential during galvanostatic conditions. The left graph shows the response of a capacitive system where the potential increases linearly. If at any point the direction of the current were reversed, the potential would also drop linearly. The middle graph shows the potential during an oxidation reaction. The potential first increases as it gets close to the formal potential. The potential will then change slowly as the oxidation reaction at $E^{0^{\prime}}$ starts. When all the molecules have been oxidized, the potential once again starts to increase. The right graph shows how the galvanostatic charging and discharging (at which the sign of the constant current is reversed) of a composite material with capacitive and redox effects might appear. This could be a mixture of carbon or conductive polymers (Chapter 4.1.3) with a redox active molecule (Chapter 2.3). 
The resulting graph shows different regimes corresponding to capacitive (black) and redox (grey) processes.
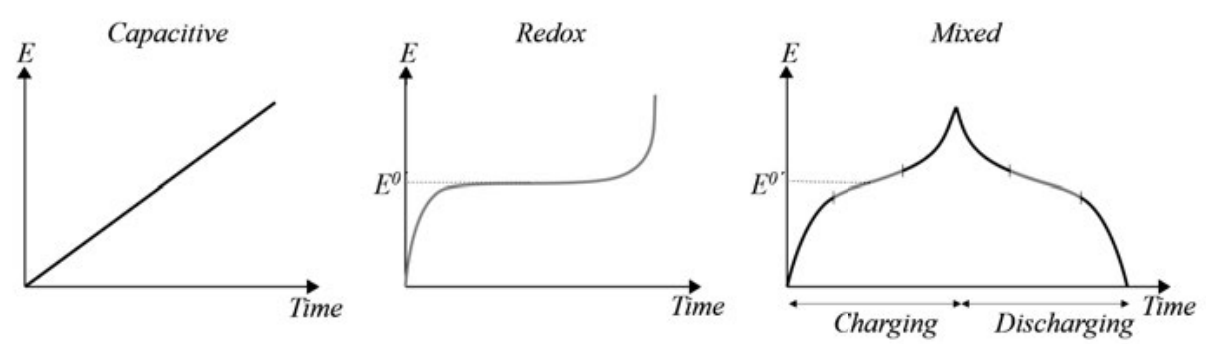

Figure 5.7 Chonopotentiometric measurements of different types of systems at constant current bias.

Chronopotentiometry is often used to measure the charge storage capacity of batteries and supercapacitors. This method is preferred over cyclic voltammetry since devices powered by such energy storage units typically operate under constant current conditions. The capacitance can be calculated by the slope $(\mathrm{dV} / \mathrm{dt}$ ) of the measurement curves according to Equation 5.2, where $I$ is the constant current. The capacitance, which is inversely proportional to the slope of the E-t curve, is a constant for the linear graph. In real systems, there is often small deviations from linearity, making capacitance a function of potential. In the redox system, the E-t curve is non-linear and capacitance will be a function of the potential. At $\mathrm{E}=E^{0^{\prime}}$ the capacitance goes to infinity as the potential remains constant since the system resembles that of an ideally non-polarizable electrode for a certain range in time. However, for these types of systems (like batteries or fuel cells) one usually doesn't talk about capacitance, but rather charge storage capacity. For hybrid (mixed) systems, it is convenient to define an average capacitance over a certain potential range $\Delta V$ and time $\Delta t$.

$$
C=\frac{d Q}{d V}=\frac{I \cdot d t}{d V}=\frac{I}{\frac{d V}{d t}}
$$

\subsubsection{Impedance Spectroscopy}

Electrical impedance $(Z)$ is the opposition of a current to flow through a circuit when a voltage is applied. The impedance is a complex value with a real and imaginary part. The real part is the well-known electrical resistance $(R)$ and the imaginary part is the reactance $(X)$ as defined in Equation 5.3 where $j$ is the 
imaginary unit and $\theta$ is the phase angle. Reactance is the opposition to the change of a voltage or current due to the inductance or capacitance of the circuit. The reactance of a capacitor and an inductor are given by Equation 5.4 where $\omega$ is the angular frequency, $C$ is the capacitance and $L$ is the inductance. Figure 5.8a gives a graphical representation of the complex impedance in complex space.

$$
\begin{aligned}
& Z=|Z| e^{j \theta}=R+j X \\
& X_{C}=-j \frac{1}{\omega C} ; X_{L}=j \omega L
\end{aligned}
$$

Unless a system is completely resistive, the impedance will be frequency dependent $(Z=Z(\omega))$. This means that a system working on an AC voltage can behave very differently depending on the frequency of the voltage. Impedance spectroscopy is a method used to characterize the impedance of a system over a range of frequencies. The impedance is measured by applying a sinusoidal AC potential, $E=E o \sin (\omega t)$, at a certain frequency and measuring the current response. In a system with linear I-E characteristic, the resulting current will also be a sinusoidal function with the form $I=I o \sin (\omega t+\theta)$, and the impedance can be calculated as $Z=E / I$. However, most systems are not linear over all frequencies. Figure $5.8 \mathrm{~b}$ shows the I-E curve of a nonlinear system. The system can be approximated as being linear over a small voltage range $d E$ around some potential $E^{*}$. By applying a small sinusoidal perturbation around this potential, the current response will also be sinusoidal to a good approximation. An impedance spectrum can be obtained by performing this type of measurement at many different frequencies. ${ }^{[145]}$

Electrochemical impedance spectroscopy (EIS) is impedance spectroscopy performed on an electrochemical cell, usually with a three-electrode setup as was described in Chapter 5.4.1. The impedance spectra can be used to investigate different physical and chemical processes happening in the cell. Since these processes often occur at different timescales, their contribution to the overall impedance of the system will vary with the frequency. By modeling the system with an equivalent circuit, where each circuit element corresponds to a certain physical process, it is possible to decouple the processes by fitting the circuit values to match the impedance spectra. Besides the values of the impedance, the phase angle gives a lot of information about what type of process is dominant at a certain frequency. A phase angle close to $0^{\circ}$ correspond to resistive behavior. This could be the resistance from ionic migration in the electrolyte or charge transfer resistance of an electrochemical reaction occurring at the electrodes. A phase angle 58 
close to $-90^{\circ}$ corresponds to capacitive behavior such as the alignment of solvent dipoles in an electric field or the formation of a double layer. In general, a resistive response is indicative of dissipation of energy while capacitive behavior indicates that energy is being stored in the system.
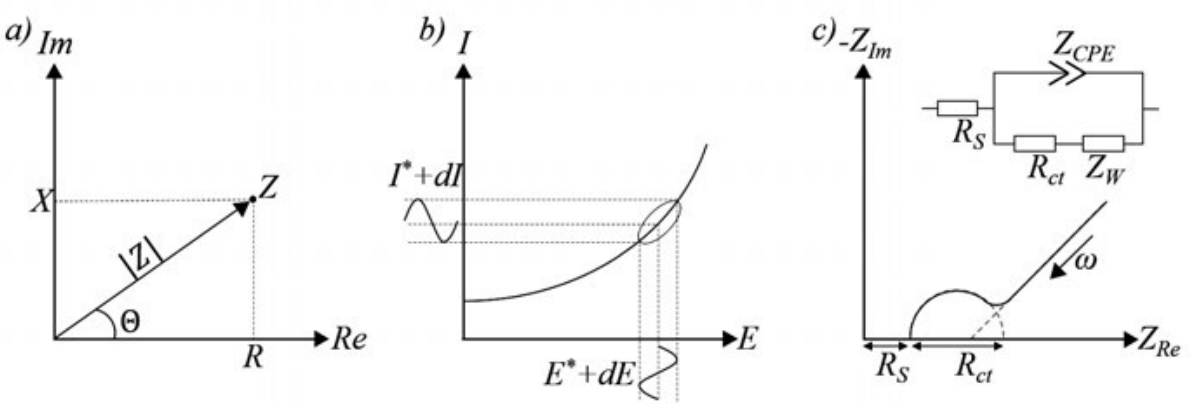

Figure 5.8 (a) Graphical representation of impedance in the complex plane, (b) the principle of quasi-linearity in impedance spectroscopy and (c) the impedance spectra of a generalized Randles equivalent circuit in a Nyquist plot with the circuit shown as an inset.

Figure 5.8c shows an impedance spectrum plotted in a Nyquist plot with the imaginary part on the $y$-axis and the real part on the $x$-axis. This spectrum has been simulated from a generalized Randles circuit (inset of Figure 5.8c), which is one of the simplest circuits used to model electrochemical systems. It consists of two resistors $\left(R_{s}\right.$ and $\left.R_{c t}\right)$, a Warburg impedance $\left(Z_{w}\right)$ and a constant phase element $\left(Z_{C P E}\right)$. The arrow shows the direction of increasing frequency. $R_{S}$ is the resistance of the electrolyte and $R_{c t}$ is the charge transfer resistance which is related to the overpotential of electrochemical reactions. The constant phase element (CPE) has the form $Z_{C P E}=1 / Q(j \omega)^{\alpha}$, where $Q$ is a constant and $\alpha$ has a value between 0 and 1 . With $\alpha=0$, the $C P E$ is a resistor with value $R=1 / Q$ and with $\alpha=1$ it equals the impedance of a capacitor with capacitance $C=Q$. In the Randles circuit, this element is usually more capacitive than resistive $(\alpha>0.5)$ and corresponds to interfacial capacitance at the electrode. For the plot in Figure 5.8c, the semicircle indicates that the $C P E$ is purely capacitive and could be replaced by a capacitor resulting in the classical Randles circuit. However, in practice the graph often shows a depressed semi-circle, indicating a deviation from purely capacitive behavior. The Warburg impedance is a CPE with $\alpha=0.5$ and corresponds to mass transport to the electrode. In the Nyquist plot of Figure 5.8c it corresponds to the $45^{\circ}$ line at low frequencies. As the electrochemical systems under investigation become more complex, so does the equivalent circuit needed to fit the data. In fact, with growing complexity, several equivalent circuits with different configurations can give the 
exact same impedance. This can lead to misinterpretation of the physical processes representing each circuit element. It is therefore good practice to keep equivalent circuits as simple as possible.[145]

\subsection{Conductivity Measurements}

Electrical conductivity $(\sigma)$ or specific conductance, measured in siemens per meter $(\mathrm{S} / \mathrm{m})$, is a material property relating the current density () passed through the material to the electric field $(E)$ when a voltage bias is applied. The conductivity of a material can be measured by the resistance $(R)$ across a cuboid with length $L$, thickness $t$ and width $w$ according to the equation shown in Figure 5.9a. However, when measuring resistance in a two-terminal setup, poor contact or injection barriers can lead to a potential drop across the interface between the probes and the material. This results in a contact resistance which will be added to the total measured resistance, and hence an underestimation of the conductivity. To avoid contact resistance, four-terminal sensing (or Kelvin sensing) is often used when measuring on highly conductive materials. The measurement involves applying a current between two points using two terminals and measuring the potential drop between an additional two terminals. With a high impedance of the measurement device, only a very small current will pass through the contacts used to measure the voltage, resulting in a negligible potential drop at these contacts. The resistance in then calculated as $R=V / I$ where $V$ is the measured voltage and $I$ is the applied current.

Figure $5.9 \mathrm{~b}$ shows how this technique can be used to measure the conductivity of thin samples of, for example, conductive polymer films. The material is coated on a substrate with four metal electrodes. The current-supplying terminals are connected to the outer electrodes while the sensing terminals are connected to the inner electrodes. If the ratio of the length between the inner electrodes and the thickness of the sample is large, then the electric field and current in the film can be thought of as evenly distributed and parallel to the film. In this situation, the same equations as for the cuboid can be used. The thickness of the sample can be measured using several different techniques such as atomic force microscopy $(A F M)$ or optical profilometry. 
a)

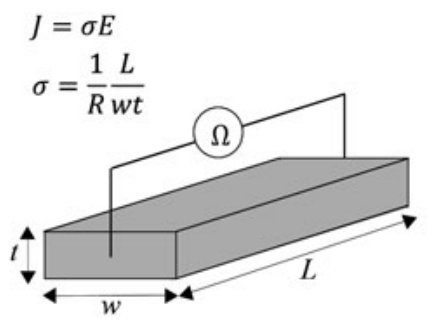

b)

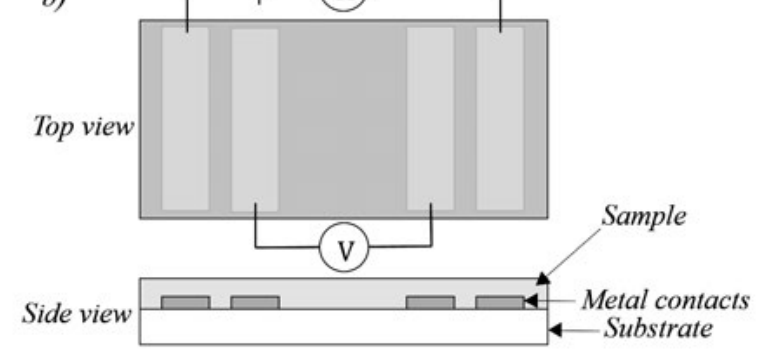

Figure 5.9 (a) Two-terminal and (b) four-terminal resistance measurements for determining electrical conductivity.

\subsection{Absorption Spectroscopy}

When electromagnetic radiation interacts with matter, it can be absorbed by the promotion of electrons to excited states of higher energy or by inducing molecular vibrations or rotation. Absorption spectroscopy is a method of determining how much light at different wavelengths is absorbed by a material. The method can be used to analyze electronic transitions in materials to learn about their electronic structure. It can also be used to investigate the composition of a compound if the absorption spectra of its constituents are known. In astronomy, astronomical spectroscopy is used to determine the composition of distant stars and interstellar gas clouds and in chemistry, absorption spectroscopy is used to analyze the composition of liquids and gases. Absorption spectroscopy can also be used to characterize optical devices like smart glass and the electrochromic properties of the materials which these devices are based on.

$$
\begin{aligned}
& \Phi=\Phi_{0} 10^{-\varepsilon c l} \\
& A=-\log _{10}(T)=-\log _{10}\left(\frac{\Phi_{0}}{\Phi}\right)=\varepsilon c l
\end{aligned}
$$

The Beer-Lambert law (Equation 5.5) relates the transmitted light flux ( $\Phi$ ) of light at a certain wavelength to the incident light flux $\left(\Phi_{0}\right)$ as it passes through a material. In Equation 5.5, $l$ is the length that light travel through the material, $c$ is the concentration of the material and $\varepsilon$ is the molar attenuation coefficient. The quotient $\Phi / \Phi_{0}$ is known as the transmittance (T). Equation 5.6 defines the absorbance $(A)$ as the negative logarithm of the transmittance. The absorbance is a useful unit since it is linear to $l, c$ and $\varepsilon$. 
The basic structure of an absorption spectrometer is shown in Figure 5.10. The light from a broad-spectrum light source is diffracted by a grating. A small opening allows the passage of a certain wavelength into the sample chamber. The transmitted light is subsequently recorded by a detector. Most spectrometers have several light sources and detectors to cover a wide range of wavelengths. By changing the angle of the diffraction grating, different wavelengths of light can be selected. An absorption spectrum is obtained by measuring the absorbance while scanning over a chosen wavelength interval. A measurement is done by first making a background spectrum of the chamber and any substrate or holder for the sample. The background spectrum will later be subtracted from the spectrum of the sample. The obtained spectrum will contain peaks with varying intensity where each peak is connected to an excitation of an energy $\Delta E=h c / \lambda$, where $h$ is the Planck constant, $c$ is the speed of light and $\lambda$ is the wavelength where the peak occurs. The intensity of the peak gives information about the relative probability of the excitation.

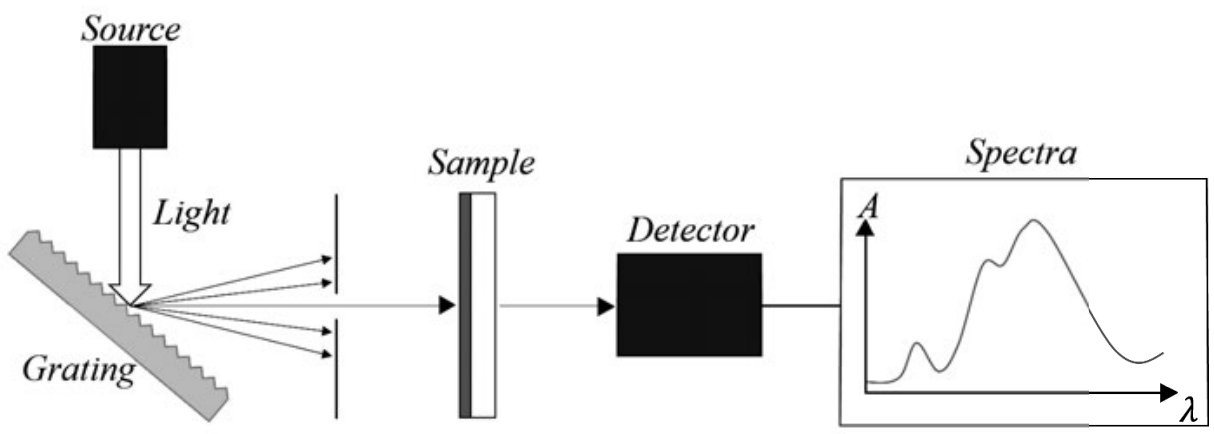

Figure 5.10 Schematic of the basic structure of an absorption spectrometer. 


\section{Conclusion and Outlook}

In this thesis, two types of flexible device technologies based on organic materials were developed. Firstly, nanofibrillated cellulose and the conductive polymer PEDOT:PSS was used to manufacture composite materials with various form factors and functionality which were further used to construct several types of electrochemical devices. Secondly, a novel method to pattern conductive polymers produced by vapor phase polymerization was developed. This technique was used to manufacture high resolution electrochromic displays on both flexible and rigid substrates.

\subsection{NFC-PEDOT:PSS Composites}

Papers I-IV deal with the development, characterization and device implementation of composites of nanofibrillated cellulose and PEDOT:PSS. In papers I, III and IV, paper-like films (nanopaper) with high conductivity were produced by solution casting while in paper II, porous aerogels were produced by freeze-drying. The motivation for using cellulose was to obtain a mechanically stable scaffold onto which PEDOT could be coated in order to realize flexible and self-supporting materials and devices.

In paper I, the nanopaper was explored as a mixed ion-electron conductor which could be used to produce "thick film" devices. The material was extensively studied to determine its microstructure as well as electronic charge transport and electrochemical properties. It was shown that PEDOT:PSS self-organize on the nanofibrils to form a uniform cladding and that all electrical and electrochemical properties remained constant when varying the thickness of the nanopapers. This enabled us to make electrochemical devices such as transistors with record high current levels and transconductance. In Paper III, the recipe of the nanopaper was adjusted to give it self-adhesive properties. This enabled us to manufacture devices by a "cut and stick"-method which we used to demonstrate the first ever logic circuits fully based on functionalized paper. We further explored the mechanical and self-adhesive properties of the nanopaper. Paper IV continues the work on paper-supercapacitors which was first explored in paper I. A redox-active molecule derived from lignin was incorporated in the nanopaper to enhance its charge storage capacity. We showed that the energy density could be significantly increased without compromising the mechanical stability, charge transport and 
power density of the nanopaper. Paper II stands out from the other papers dealing with cellulose composites in that bulky porous structures were manufactured instead of nanopapers. These aerogels had a superior flexibility compared to the nanopapers and could reversibly be compressed by more than $90 \%$. The compression resulted in a change in the resistance of the of the material, and this property made it possible to use the aerogels as resistive pressure sensors. Furthermore, due to the high Seebeck coefficient of PEDOT:PSS, a thermovoltage induced by a temperature gradient across the material could be registered. This enabled us to use the aerogels as dual-parameter sensors to simultaneously measure pressure and temperature.

Two common denominators for all cellulose composites are flexibility and scalability. The flexibility, self-adhesiveness and "softness" of the nanopapers and aerogels developed in papers II and III could find possible applications as electronic skin and wearable electronics. These materials have already been used as contacting electrodes to biological environments in the new field of plantbioelectronics, and the next step could be to use them on humans to measure physiological parameters such as skin conductance, pulse and temperature changes. The supercapacitors developed in paper I and then further improved in paper IV hold great promise as a green technology for energy storage. The hope is that these materials in the future can be produced in large scale by a manufacturing technique similar to modern papermaking. However, a number of technical details need to be addressed in order to reach this level of upscaling in production which will require further research and development. Furthermore, the current price of commercial PEDOT:PSS is a limiting factor which needs to be overcome to be able to compete with supercapacitors and batteries on the market.

\subsection{Patterning Conductive Polymers with UV Light}

There are numerous ways of patterning organic materials on flexible substrates to make electronic devices and circuits. Printing methods such as screen printing and inkjet printing have been frequently used in the field of printed electronics to manufacture devices at high throughput and large area. However, these techniques are often lacking in resolution, and so to fabricate devices with small dimensions, researchers still rely on traditional patterning methods like photolithography for many applications. However, photolithography has many shortcomings when it comes to patterning organic materials, such as many patterning steps and degradation of the organic material due to hazardous 64 
solvents. We developed a patterning method for conductive polymers produced by vapor phase polymerization which, much like photolithography, uses UV light to define a pattern. However, this is done without the use of a photoresist and other associated chemicals. The technique was introduced and explored in paper $\mathrm{V}$ where we showed that it could be used, both to pattern the conductive polymer PEDOT:Tos with a "step-like" profile or to locally control the conductivity in a continuous film. We also demonstrated how the optical properties of the polymer could be tuned and this was used to pattern high resolution images with optical gradients. Furthermore, the patterning process as well as the resulting material was extensively investigated to better understand the mechanism behind the technique. In paper VI we demonstrated that the technique could also be used on the conductive polymer PPy which has complementary electrochromic properties to PEDOT. Different dynamic electrochromic devices were demonstrated on both flexible plastic substrates as well as rigid glass substrates.

The method is an interesting alternative to photolithography and other patterning techniques. However, the patterning resolution is currently in the order of $10 \mu \mathrm{m}$, and to truly become an alternative to photolithography, sub-micrometer resolution must be achieved. In our investigations, we did not encounter any theoretical/fundamental limitation to the resolution of the technique, so with further optimization and understanding of the patterning process we hope that this goal can be reached. However, the technique goes beyond mere patterning by adding the possibility of spatially controlling the optical and electrical properties of conductive polymer films in a continuous way. Besides the applications already explored such as electrochromic devices, we believe that such control over material properties could lead to the development of new metamaterials and other functional materials. 


\section{References}

[1] H. Letheby, Journal of the Chemical Society 1862, 15, 161.

[2] H. Shirakawa, E. J. Louis, A. G. MacDiarmid, C. K. Chiang, A. J. Heeger, Journal of the Chemical Society, Chemical Communications 1977, 578.

[3] A. O. Patil, A. J. Heeger, F. Wudl, Chemical Reviews 1988, 88, 183.

[4] S. Kera, N. Ueno, Journal of Electron Spectroscopy and Related Phenomena 2015, 204, Part A, 2.

[5] A. Elschner, PEDOT : principles and applications of an intrinsically conductive polymer, CRC Press, Boca Raton, FL 2011.

[6] J. Kawahara, P. A. Ersman, I. Engquist, M. Berggren, Organic Electronics: physics, materials, applications 2012, 13, 469.

[7] C. Karlsson, J. Nicholas, D. Evans, M. Forsyth, M. Strømme, M. Sjödin, P. C. Howlett, C. Pozo-Gonzalo, ChemSusChem 2016, 9, 2112.

[8] D. Evans, M. Fabretto, M. Mueller, K. Zuber, R. Short, P. J. Murphy, Journal of Materials Chemistry 2012, 22, 14889.

[9] M. N. Gueye, A. Carella, N. Massonnet, E. Yvenou, S. Brenet, J. Faure-Vincent, S. Pouget, F. Rieutord, H. Okuno, A. Benayad, R. Demadrille, J.-P. Simonato, Chemistry of Materials 2016, 28, 3462.

[10] O. Bubnova, Z. U. Khan, H. Wang, S. Braun, D. R. Evans, M. Fabretto, P. HojatiTalemi, D. Dagnelund, J.-B. Arlin, Y. H. Geerts, S. Desbief, D. W. Breiby, J. W. Andreasen, R. Lazzaroni, W. M. Chen, I. Zozoulenko, M. Fahlman, P. J. Murphy, M. Berggren, X. Crispin, Nat Mater 2014, 13, 190.

[11] A. G. MacDiarmid, A. J. Epstein, Synthetic Metals 1994, 65, 103.

[12] J. Nevrela, M. Micjan, M. Novota, S. Kovacova, M. Pavuk, P. Juhasz, J. Kovac, J. Jakabovic, M. Weis, Journal of Polymer Science Part B: Polymer Physics 2015, 53, 1139.

[13] A. M. Nardes, R. A. J. Janssen, M. Kemerink, Advanced Functional Materials 2008, 18, 865 .

[14] X. Crispin, F. L. E. Jakobsson, A. Crispin, P. C. M. Grim, P. Andersson, A. Volodin, C. van Haesendonck, M. Van der Auweraer, W. R. Salaneck, M. Berggren, Chemistry of Materials 2006, 18, 4354.

[15] S. Timpanaro, M. Kemerink, F. J. Touwslager, M. M. De Kok, S. Schrader, Chemical Physics Letters 2004, 394, 339.

[16] C. Ionescu-Zanetti, A. Mechler, S. A. Carter, R. Lal, Advanced Materials 2004, 16, 385.

[17] D. Klemm, F. Kramer, S. Moritz, T. Lindström, M. Ankerfors, D. Gray, A. Dorris, Angewandte Chemie International Edition 2011, 50, 5438.

[18] H. Zhu, Z. Jia, Y. Chen, N. Weadock, J. Wan, O. Vaaland, X. Han, T. Li, L. Hu, Nano Letters 2013, 13, 3093.

[19] M. T. Islam, M. M. Alam, A. Patrucco, A. Montarsolo, M. Zoccola, AATCC Journal of Research 2014, 1, 17.

[20] S. H. Osong, S. Norgren, P. Engstrand, Cellulose 2016, 23, 93. 
[21] M. Henriksson, L. A. Berglund, P. Isaksson, T. Lindström, T. Nishino, Biomacromolecules 2008, 9, 1579.

[22] F. Hoeng, A. Denneulin, J. Bras, Nanoscale 2016, 8, 13131.

[23] M. G. Hayes, A. L. Kelly, Journal of Dairy Research 2003, 70, 297.

[24] S. Kalia, S. Boufi, A. Celli, S. Kango, Colloid and Polymer Science 2014, 292, 5.

[25] S. P. Mishra, A. S. Manent, B. Chabot, C. Daneault, BioResources 2012, 7, 422.

[26] A. Tejado, M. N. Alam, M. Antal, H. Yang, T. G. M. van de Ven, Cellulose 2012, $19,831$.

[27] A. Isogai, T. Saito, H. Fukuzumi, Nanoscale 2011, 3, 71.

[28] K. M. O. Håkansson, A. B. Fall, F. Lundell, S. Yu, C. Krywka, S. V. Roth, G. Santoro, M. Kvick, L. Prahl Wittberg, L. Wågberg, L. D. Söderberg, Nature Communications 2014, 5, 4018.

[29] S. Ma, Q. Mi, J. Yu, J. He, J. Zhang, Progress in Chemistry 2014, 26, 796.

[30] E. Cuce, P. M. Cuce, C. J. Wood, S. B. Riffat, Renewable and Sustainable Energy Reviews 2014, 34, 273.

[31] J. Stergar, U. Maver, Journal of Sol-Gel Science and Technology 2016, 77, 738.

[32] M. Hamedi, E. Karabulut, A. Marais, A. Herland, G. Nyström, L. Wågberg, Angewandte Chemie International Edition 2013, 52, 12038.

[33] G. Milczarek, O. Inganäs, Science 2012, 335, 1468.

[34] P. Navalpotro, J. Palma, M. Anderson, R. Marcilla, Journal of Power Sources 2016, 306, 711.

[35] G. L. Soloveichik, Chemical Reviews 2015, 115, 11533.

[36] M. Pandurangappa, N. S. Lawrence, R. G. Compton, Analyst 2002, 127, 1568.

[37] C. Karlsson, H. Huang, M. Strømme, A. Gogoll, M. Sjödin, Journal of Electroanalytical Chemistry 2014, 735, 95.

[38] C. Karlsson, H. Huang, M. Stromme, A. Gogoll, M. Sjodin, RSC Advances 2015, 5, 11309.

[39] F. N. Ajjan, N. Casado, T. Rębiś, A. Elfwing, N. Solin, D. Mecerreyes, O. Inganäs, J. Mater. Chem. A 2016, 4, 1838.

[40] T. Y. Nilsson, M. Wagner, O. Inganäs, ChemSusChem 2015, 8, 4081.

[41] N. Casado, M. Hilder, C. Pozo-Gonzalo, M. Forsyth, D. Mecerreyes, ChemSusChem 2017, 10, 1.

[42] T. Rębiś, G. Milczarek, Electrochimica Acta 2016, 204, 108.

[43] S. Admassie, F. N. Ajjan, A. Elfwing, O. Inganas, Materials Horizons 2016, 3, 174.

[44] D. H. Nagaraju, T. Rebis, R. Gabrielsson, A. Elfwing, G. Milczarek, O. Inganäs, Advanced Energy Materials 2014, 4.

[45] P. Andersson Ersman, D. Nilsson, J. Kawahara, G. Gustafsson, M. Berggren, Organic Electronics: physics, materials, applications 2013, 14, 1276.

[46] J. Kawahara, P. Andersson Ersman, D. Nilsson, K. Katoh, Y. Nakata, M. Sandberg, M. Nilsson, G. Gustafsson, M. Berggren, Journal of Polymer Science, Part B: Polymer Physics 2013, 51, 265.

[47] E. Quartarone, P. Mustarelli, A. Magistris, Solid State lonics 1998, 110, 1.

[48] S. Baldelli, Accounts of Chemical Research 2008, 41, 421. 
[49] Y. Lauw, M. D. Horne, T. Rodopoulos, A. Nelson, F. A. M. Leermakers, The Journal of Physical Chemistry B 2010, 114, 11149.

[50] T. Romann, O. Oll, P. Pikma, K. Kirsimäe, E. Lust, Journal of Power Sources 2015, 280, 606.

[51] S. Kazemiabnavi, Z. Zhang, K. Thornton, S. Banerjee, The Journal of Physical Chemistry B 2016, 120, 5691.

[52] A. Jarosik, S. R. Krajewski, A. Lewandowski, P. Radzimski, Journal of Molecular Liquids 2006, 123, 43.

[53] C. Largeot, C. Portet, J. Chmiola, P.-L. Taberna, Y. Gogotsi, P. Simon, Journal of the American Chemical Society 2008, 130, 2730.

[54] L. Chen, M. Sharifzadeh, N. Mac Dowell, T. Welton, N. Shah, J. P. Hallett, Green Chemistry 2014, 16, 3098.

[55] H. Sirringhaus, T. Kawase, R. H. Friend, T. Shimoda, M. Inbasekaran, W. Wu, E. P. Woo, Science 2000, 290, 2123.

[56] D. Nilsson, N. Robinson, M. Berggren, R. Forchheimer, Advanced Materials 2005, 17, 353.

[57] P. Kopola, M. Tuomikoski, R. Suhonen, A. Maaninen, Thin Solid Films 2009, 517, 5757.

[58] D. A. Pardo, G. E. Jabbour, N. Peyghambarian, Advanced Materials 2000, 12, 1249.

[59] S. C. Chang, J. Liu, J. Bharathan, Y. Yang, J. Onohara, J. Kido, Advanced Materials 1999, 11, 734.

[60] R. Søndergaard, M. Hösel, D. Angmo, T. T. Larsen-Olsen, F. C. Krebs, Materials Today 2012, 15, 36.

[61] T. M. Eggenhuisen, Y. Galagan, A. F. K. V. Biezemans, T. M. W. L. Slaats, W. P. Voorthuijzen, S. Kommeren, S. Shanmugam, J. P. Teunissen, A. Hadipour, W. J. H. Verhees, S. C. Veenstra, M. J. J. Coenen, J. Gilot, R. Andriessen, W. A. Groen, J. Mater. Chem. A 2015, 3, 7255.

[62] H. J. Lee, T. H. Park, J. H. Choi, E. H. Song, S. J. Shin, H. Kim, K. C. Choi, Y. W. Park, B. K. Ju, Organic Electronics: physics, materials, applications 2013, 14, 416.

[63] C. D. O'Connell, M. J. Higgins, H. Nakashima, S. E. Moulton, G. G. Wallace, Langmuir 2012, 28, 9953.

[64] J. A. Defranco, B. S. Schmidt, M. Lipson, G. G. Malliaras, Organic Electronics: physics, materials, applications 2006, 7, 22.

[65] J. Huang, R. Xia, Y. Kim, X. Wang, J. Dane, O. Hofmann, A. Mosley, A. J. de Mello, J. C. de Mello, D. D. C. Bradley, Journal of Materials Chemistry 2007, 17, 1043.

[66] P. G. Taylor, J. K. Lee, A. A. Zakhidov, M. Chatzichristidi, H. H. Fong, J. A. DeFranco, G. G. Malliaras, C. K. Ober, Advanced Materials 2009, 21, 2314.

[67] J. Kim, J. You, E. Kim, Macromolecules 2010, 43, 2322.

[68] D. Tobjörk, R. Österbacka, Advanced Materials 2011, 23, 1935.

[69] L. Nyholm, G. Nyström, A. Mihranyan, M. Strømme, Advanced Materials 2011, 23, 3751. 
[70] M. Agarwal, Q. Xing, B. S. Shim, N. Kotov, K. Varahramyan, Y. Lvov, Nanotechnology 2009, 20.

[71] M. M. Hamedi, A. Hajian, A. B. Fall, K. Håkansson, M. Salajkova, F. Lundell, L. Wågberg, L. A. Berglund, ACS Nano 2014, 8, 2467.

[72] B. Andres, S. Forsberg, C. Dahlström, N. Blomquist, H. Olin, physica status solidi (b) 2014, 251, 2581.

[73] C. Sasso, E. Zeno, M. Petit-Conil, D. Chaussy, M. N. Belgacem, S. Tapin-Lingua, D. Beneventi, Macromolecular Materials and Engineering 2010, 295, 934.

[74] B. Anothumakkool, R. Soni, S. N. Bhange, S. Kurungot, Energy and Environmental Science 2015, 8, 1339.

[75] J. Kawahara, P. Andersson Ersman, X. Wang, G. Gustafsson, H. Granberg, M. Berggren, Organic Electronics: physics, materials, applications 2013, 14, 3061.

[76] X. Qian, J. Shen, G. Yu, X. An, BioResources 2010, 5, 899.

[77] N. Sani, X. Wang, H. Granberg, P. Andersson Ersman, X. Crispin, P. Dyreklev, I. Engquist, G. Gustafsson, M. Berggren, Scientific Reports 2016, 6, 28921.

[78] M. Nogi, M. Karakawa, N. Komoda, H. Yagyu, T. T. Nge, Scientific Reports 2015, 5, 17254 .

[79] G. Nyström, A. Razaq, M. Strømme, L. Nyholm, A. Mihranyan, Nano Letters 2009, 9, 3635.

[80] X. Chen, H. Zhu, C. Liu, Y. C. Chen, N. Weadock, G. Rubloff, L. Hu, J. Mater. Chem. A 2013, 1, 8201.

[81] Z. Gui, H. Zhu, E. Gillette, X. Han, G. W. Rubloff, L. Hu, S. B. Lee, ACS Nano 2013, 7, 6037.

[82] S. Leijonmarck, A. Cornell, G. Lindbergh, L. Wågberg, J. Mater. Chem. A 2013, $1,4671$.

[83] K. B. Lee, Journal of Micromechanics and Microengineering 2005, 15, S210.

[84] Q. Cheng, Z. Song, T. Ma, B. B. Smith, R. Tang, H. Yu, H. Jiang, C. K. Chan, Nano Letters 2013, 13, 4969.

[85] Q. Nguyen, B. H. Kim, J. W. Kwon, Journal of Microelectromechanical Systems 2015, 24, 519.

[86] C. Wu, X. Wang, L. Lin, H. Guo, Z. L. Wang, ACS Nano 2016, 10, 4652.

[87] J. P. Rojas, D. Conchouso, A. Arevalo, D. Singh, I. G. Foulds, M. M. Hussain, Nano Energy 2017, 31, 296.

[88] M. Nogi, M. Karakawa, N. Komoda, H. Yagyu, T. T. Nge, Scientific Reports 2015, 5.

[89] A. K. Yetisen, M. S. Akram, C. R. Lowe, Lab on a Chip - Miniaturisation for Chemistry and Biology 2013, 13, 2210.

[90] D. M. Cate, J. A. Adkins, J. Mettakoonpitak, C. S. Henry, Analytical Chemistry 2015, 87, 19.

[91] W. Dungchai, O. Chailapakul, C. S. Henry, Analytical Chemistry 2009, 81, 5821.

[92] E. Carrilho, A. W. Martinez, G. M. Whitesides, Analytical Chemistry 2009, 81, 7091.

[93] K. Abe, K. Suzuki, D. Citterio, Analytical Chemistry 2008, 80, 6928. 
[94] A. W. Martinez, S. T. Phillips, M. J. Butte, G. M. Whitesides, Angewandte Chemie International Edition 2007, 46, 1318.

[95] M. M. Hamedi, A. Ainla, F. Güder, D. C. Christodouleas, M. T. FernándezAbedul, G. M. Whitesides, Advanced Materials 2016, 28, 5054.

[96] H. D. Wang, U. D. Ail, R. D. Gabrielsson, M. P. Berggren, X. Crispin, Advanced Energy Materials 2015, 5.

[97] X. Ren, P. G. Pickup, Journal of the Chemical Society, Faraday Transactions 1993, 89, 321.

[98] G. L. Duffitt, P. G. Pickup, Journal of the Chemical Society, Faraday Transactions 1992, 88, 1417.

[99] Z. Yu, L. Tetard, L. Zhai, J. Thomas, Energy \& Environmental Science 2015, 8, 702.

[100] G. Wee, O. Larsson, M. Srinivasan, M. Berggren, X. Crispin, S. Mhaisalkar, Advanced Functional Materials 2010, 20, 4344.

[101] Z. Wu, Y. Zhu, X. Ji, J. Mater. Chem. A 2014, 2, 14759.

[102] G. Wang, L. Zhang, J. Zhang, Chemical Society Reviews 2012, 41, 797.

[103] R. Kötz, M. Carlen, Electrochimica Acta 2000, 45, 2483.

[104] V. V. N. Obreja, A. Dinescu, A. C. Obreja, International Review of Electrical Engineering 2010, 5, 272.

[105] P. Simon, Y. Gogotsi, Nature Materials 2008, 7, 845.

[106] M. Winter, R. J. Brodd, Chemical Reviews 2004, 104, 4245.

[107] A. Vlad, N. Singh, J. Rolland, S. Melinte, P. M. Ajayan, J. F. Gohy, Scientific Reports 2014, 4, 4315.

[108] F. Zhang, T. Zhang, X. Yang, L. Zhang, K. Leng, Y. Huang, Y. Chen, Energy \& Environmental Science 2013, 6, 1623.

[109] M. Wagner, T. Rębiś, O. Inganäs, Journal of Power Sources 2016, 302, 324.

[110] C. M. Proctor, J. Rivnay, G. G. Malliaras, Journal of Polymer Science, Part B: Polymer Physics 2016, 54, 1433.

[111] D. Khodagholy, J. Rivnay, M. Sessolo, M. Gurfinkel, P. Leleux, L. H. Jimison, E. Stavrinidou, T. Herve, S. Sanaur, R. M. Owens, G. G. Malliaras, Nat Commun 2013, 4.

[112] J. Rivnay, P. Leleux, M. Ferro, M. Sessolo, A. Williamson, D. A. Koutsouras, D. Khodagholy, M. Ramuz, X. Strakosas, R. M. Owens, C. Benar, J.-M. Badier, C. Bernard, G. G. Malliaras, in Sci Adv, Vol. 1, 2015, e1400251.

[113] J.-P. Fleurial, JOM 2009, 61, 79.

[114] D. Tatarinov, D. Wallig, G. Bastian, Journal of Electronic Materials 2012, 41, 1706.

[115] B. Poudel, Q. Hao, Y. Ma, Y. Lan, A. Minnich, B. Yu, X. Yan, D. Wang, A. Muto, D. Vashaee, X. Chen, J. Liu, M. S. Dresselhaus, G. Chen, Z. Ren, Science 2008, 320, 634.

[116] R. Amatya, R. J. Ram, Journal of electronic materials 2012, 41, 1011.

[117] C. Han, Z. Li, S. Dou, Chinese Science Bulletin 2014, 59, 2073.

[118] D. Briand, F. Molina-Lopez, A. V. Quintero, C. Ataman, J. Courbat, N. F. de Rooij, Procedia Engineering 2011, 25, 8. 
[119] C. Wu, T. W. Kim, F. Li, T. Guo, ACS Nano 2016, 10, 6449.

[120] T. J. Seebeck, Abhandlungen der Deutschen Akademie der Wissenschaften zu Berlin 1822, 289.

[121] C. M. Lampert, Solar Energy Materials 1984, $11,1$.

[122] P. Andersson, R. Forchheimer, P. Tehrani, M. Berggren, Advanced Functional Materials 2007, 17, 3074.

[123] P. Andersson Ersman, J. Kawahara, M. Berggren, Organic Electronics: physics, materials, applications 2013, 14, 3371.

[124] A. F. Diaz, K. K. Kanazawa, G. P. Gardini, Journal of the Chemical Society, Chemical Communications 1979, 635.

[125] R. E. Myers, Journal of Electronic Materials 1986, 15, 61.

[126] B. Winther-Jensen, K. West, Macromolecules 2004, 37, 4538.

[127] P. A. Levermore, L. Chen, X. Wang, R. Das, D. D. C. Bradley, Advanced Materials 2007, 19, 2379.

[128] M. V. Fabretto, D. R. Evans, M. Mueller, K. Zuber, P. Hojati-Talemi, R. D. Short, G. G. Wallace, P. J. Murphy, Chemistry of Materials 2012, 24, 3998.

[129] B. Winther-Jensen, J. Chen, K. West, G. Wallace, Macromolecules 2004, 37, 5930.

[130] S. Admassie, F. Zhang, A. G. Manoj, M. Svensson, M. R. Andersson, O. Inganäs, Solar Energy Materials and Solar Cells 2006, 90, 133.

[131] B. Cho, K. S. Park, J. Baek, H. S. Oh, Y.-E. Koo Lee, M. M. Sung, Nano Letters 2014, 14, 3321.

[132] M. Fabretto, K. Zuber, C. Hall, P. Murphy, H. J. Griesser, Journal of Materials Chemistry 2009, 19, 7871.

[133] M. Mueller, M. Fabretto, D. Evans, P. Hojati-Talemi, C. Gruber, P. Murphy, Polymer 2012, 53, 2146.

[134] R. Brooke, M. Fabretto, P. Hojati-Talemi, P. Murphy, D. Evans, Polymer 2014, 55, 3458.

[135] A. El Kadib, M. Bousmina, Chemistry - A European Journal 2012, 18, 8264.

[136] H. Dong, J. F. Snyder, D. T. Tran, J. L. Leadore, Carbohydrate Polymers 2013, 95, 760.

[137] Y. Sun, M. Yang, F. Yu, J. Chen, J. Ma, Progress in Chemistry 2015, 27, 1133.

[138] J. Y. Hong, E. H. Sohn, S. Park, H. S. Park, Chemical Engineering Journal 2015, 269, 229.

[139] R. Baetens, B. P. Jelle, A. Gustavsen, Energy and Buildings 2011, 43, 761.

[140] M. Koebel, A. Rigacci, P. Achard, Journal of Sol-Gel Science and Technology 2012, 63, 315.

[141] X. Wang, L. Liu, X. Wang, L. Bai, H. Wu, X. Zhang, L. Yi, Q. Chen, Journal of Solid State Electrochemistry 2011, 15, 643.

[142] H. Gholipour-Ranjbar, M. R. Ganjali, P. Norouzi, H. R. Naderi, Journal of Materials Science: Materials in Electronics 2016, 27, 10163.

[143] Z. Yu, M. McInnis, J. Calderon, S. Seal, L. Zhai, J. Thomas, Nano Energy 2015, 11, 611. 
[144] K. S. Sohn, S. J. Oh, E. J. Kim, J. M. Gim, N. S. Kim, Y. S. Kim, J. W. Kim, Transactions on Electrical and Electronic Materials 2013, 14, 273.

[145] X.-Z. Yuan, C. Song, H. Wang, J. Zhang, Springer, Belgium, Europe 2010. 
Part II

Papers 
